\title{
Point-Substitution of Phenylalanine Residues of 26RFa Neuropeptide: A Structure-Activity Relationship Study
}

\author{
Benjamin Lefranc ${ }^{1,2,+} \oplus$, Karima Alim ${ }^{1,+}$, Cindy Neveu ${ }^{1,+}$, Olivier Le Marec ${ }^{1,+}$, Christophe Dubessy ${ }^{1,2}\left({ }^{\circ}\right.$, \\ Jean A. Boutin ${ }^{3,4}{ }^{(}$, Julien Chuquet ${ }^{1}{ }^{1}$, David Vaudry ${ }^{1,2}$, Gaëtan Prévost ${ }^{1}$, Marie Picot ${ }^{1}$, Hubert Vaudry ${ }^{1,2}$, \\ Nicolas Chartrel ${ }^{1}$ and Jérôme Leprince ${ }^{1,2, * \mathbb{D}}$
}

1 INSERM U1239, Laboratory of Neuronal and Neuroendocrine Differentiation and Communication, Normandy University, 76000 Rouen, France; benjamin.lefranc@univ-rouen.fr (B.L.); alimkarima8@gmail.com (K.A.); duchemin@neurofit.com (C.N.); lemarec.olivier@gmail.com (O.L.M.); christophe.dubessy@univ-rouen.fr (C.D.); julien.chuquet@univ-rouen.fr (J.C.); david.vaudry@univ-rouen.fr (D.V.); gaetan.prevost@chu-rouen.fr (G.P.); marie.picot@univ-rouen.fr (M.P.); hubert.vaudry@univ-rouen.fr (H.V.); nicolas.chartrel@univ-rouen.fr (N.C.)

2 Cell Imaging Platform of Normandy, PRIMACEN, Normandy University, 76000 Rouen, France

3 Institut de Recherches Internationales SERVIER, 50 rue Carnot, 92284 Suresnes, France; ja.boutin.pro@gmail.com

4 PHARMADEV, Faculté de Pharmacie, Université de Toulouse, 31062 Toulouse, France

* Correspondence: jerome.leprince@univ-rouen.fr; Tel.: +33-235-14-6132

+ These authors equally contributed to this work.

\section{check for} updates

Citation: Lefranc, B.; Alim, K.; Neveu, C.; Le Marec, O.; Dubessy, C.; Boutin, J.A.; Chuquet, J.; Vaudry, D.; Prévost, G.; Picot, M.; et al.

Point-Substitution of Phenylalanine Residues of 26RFa Neuropeptide: A Structure-Activity Relationship Study. Molecules 2021, 26, 4312. https:// doi.org/10.3390/molecules26144312

Academic Editor:

Katalin Prokai-Tatrai

Received: 7 June 2021

Accepted: 12 July 2021

Published: 16 July 2021

Publisher's Note: MDPI stays neutral with regard to jurisdictional claims in published maps and institutional affiliations.

Copyright: (C) 2021 by the authors. Licensee MDPI, Basel, Switzerland. This article is an open access article distributed under the terms and conditions of the Creative Commons Attribution (CC BY) license (https:/ / creativecommons.org/licenses/by/ $4.0 /)$.
Abstract: 26RFa is a neuropeptide that activates the rhodopsin-like G protein-coupled receptor QRFPR/GPR103. This peptidergic system is involved in the regulation of a wide array of physiological processes including feeding behavior and glucose homeostasis. Herein, the pharmacological profile of a homogenous library of QRFPR-targeting peptide derivatives was investigated in vitro on human QRFPR-transfected cells with the aim to provide possible insights into the structural determinants of the Phe residues to govern receptor activation. Our work advocates to include in next generations of $26 \mathrm{RFa}_{(20-26)}$-based QRFPR agonists effective substitutions for each Phe unit, i.e., replacement of the $\mathrm{Phe}^{22}$ residue by a constrained 1,2,3,4-tetrahydroisoquinoline-3-carboxylic acid moiety, and substitution of both $\mathrm{Phe}^{24}$ and $\mathrm{Phe}^{26}$ by their para-chloro counterpart. Taken as a whole, this study emphasizes that optimized modifications in the C-terminal part of 26RFa are mandatory to design selective and potent peptide agonists for human QRFPR.

Keywords: GPR103; QRFP; RFamide; intracellular calcium concentration; peptide analog

\section{Introduction}

The neuropeptides 26RFa (QRFP26) and its N-terminal extended form 43RFa (QRFP, Figure 1) are the endogenous ligands of the $G_{i / s / q}$ coupled QRFP receptor (QRFPR), formerly known as the orphan receptor GPR103 [1-3]. Since the isolation of 26RFa from a European green frog Rana esculenta brain extract [4], prepro-26RFa cDNA has been characterized in numerous genomes of diverse vertebrates from fish to mammals [5]. However, very few mature peptides have been identified so far, leaving the 26RFa/QRFP precursor post-translational processing uncertain. Indeed, in avians, 26RFa orthologs have been isolated and sequenced from the Japanese quail [6] and zebra finch [7] while, in rodents, only the $43 \mathrm{RFa}$ counterpart has been characterized from rat $[2,8]$. In humans, both 26RFa- and 43RFa-immunoreactive forms have been detected in hypothalamic and spinal cord extracts [9]. Interestingly, the presence of a tribasic cleavage site (Arg-Lys-Arg/Lys) within the 26RFa sequence suggests that the C-terminal heptapeptide GGFSFRF-NH ${ }_{2}$, named $26 \mathrm{RFa}_{(20-26)}$, could also be produced from the precursor. However, to date, this fragment, whose sequence is highly conserved in tetrapods, has not been identified as a mature product of the 26RFa/QRFP gene transcription. 
Human 26RFa:

TSGPLGNLAEELNGYSRKKGGFSFRF-NH $\mathrm{NH}_{2}$

\section{Human 43RFa: \\ <EDEGSEATGFLPAAGEKTSGPLGNLAEELNGYSRKKGGFSFRF-NH $\mathrm{N}_{2}$}

Figure 1. Primary structures of human 26RFa and 43RFa/QRFP. $<$ E denotes pyroglutamic acid.

In rats, the $26 \mathrm{RFa} / \mathrm{QRFP}$ gene is primarily expressed in hypothalamic neurons which project in various brain areas $[1,2,8,10]$. Consistent with the widespread distribution of 26RFa/QRFP-containing fibers, 26RFa and/or QRFP are involved in the regulation of multiple physiological activities [5]. In particular, it is now firmly established that 26RFa and QRFP stimulate food intake in rodents $[1,8,10-16]$. There is also evidence that $26 \mathrm{RFa}$ and/or QRFP regulate several other neuroendocrine and cognitive functions including reproduction [17,18], anxiety [19], memory [20], cardiovascular activity [8] and nociceptive transmission [21-23], some of which depend, at least in part, on off-target interaction with QRFPR-related receptors [24,25]. In addition, recent data reveal that the 26RFa/QRFPR system controls glucose homeostasis at the periphery by increasing insulin sensitivity and inhibiting hepatic glucose production [26-29]. Thus, QRFPR positions as an attractive target for the development of innovative drugs. Indeed, there is now clinical evidence for possible indications of QRFPR ligands for the treatment of metabolic disorders, obesity and diabetes [5].

As a molecular signature, the RF-amide C-terminal extremity of 26RFa/QRFP and other RF-amide-related peptides (i.e., NPAF/NPFF, PrRP, RFRP-1(GnIH)/RFRP-3 and kisspeptin in humans [30]) represents the chemical determinant for bioactivity. Notably, deamidation or local side-chain substitution of the RF-amide moiety is generally associated with full or partial loss of activity and/or affinity of these neuropeptides [2,31-33]. The $\mathrm{N}$-terminal part of RF-amide-related peptides diverges in their sequence and length, and conditions the whole or partial affinity and selectivity for one or several receptors, respectively [34,35]. For instance, 26RFa also binds with nanomolar affinity to both NPFF1 and NPFF2 receptors [24]. However, 26RFa can be downsized from the N-terminal extremity with a gradual decrease in its ability to mobilize intracellular calcium concentration $\left(\left[\mathrm{Ca}^{2+}\right]_{\mathrm{i}}\right)$ in CHO cells transfected by the human QRFPR ( $h$ QRFPR) [36]. Since $26 \mathrm{RFa}_{(20-26)}(\mathrm{LV}-2021)$ mimics the orexigenic and gonadotropic effects of full-length 26RFa [12,17], it appears that this C-terminal heptapeptide can serve as a molecular scaffold for designing low molecular weight, potent and stable $h \mathrm{QRFPR}$ ligands. As a matter of fact, $26 \mathrm{RFa}_{(20-26)}$ has already been successfully used to design nanomolar active agonists such as $\left[\mathrm{Nva}^{23}\right] 26 \mathrm{RFa}{ }_{(20-26)}(\mathrm{LV}-$ 2073) [36], [Cmpi ${ }^{21}$, aza- $\left.\beta^{3}-\mathrm{Hht}^{23}\right] 26 \mathrm{RFa}_{(21-26)}(\mathrm{LV}-2172)$ [37], and [(Me) $\left.{ }^{\omega} \mathrm{Arg}^{25}\right] 26 \mathrm{RFa}(20-26)$ (LV-2186) [38], as well as proteolytic resistant pseudopeptides (Figure 2) [39]. According to our previous structure-activity relationship studies [for review, 5], these compounds exhibit modifications at positions occupied by residues classified as permissive to substitution or engaged in peptide bonds susceptible to degradation. In contrast, the residues of the $-\mathrm{Phe}^{24}-\mathrm{Arg}^{25}-\mathrm{Phe}^{26}-\mathrm{NH}_{2}$ triad are very sensitive to substitutions and only tolerate subtle amendments for improving bioactivity like the $\omega$-methylation of arginine 25 [38]. To date, few replacements have been reported in the phenylalanine positions of $26 \mathrm{RFa}_{(20-26)}$ [5]. As previously suggested for PrRP and kisspeptin [40], our work emphasizes that optimized modifications in the $\mathrm{C}$-terminal part of $26 \mathrm{RFa}$ are mandatory to design selective and potent peptidergic ligands for $h$ QRFPR. 
A

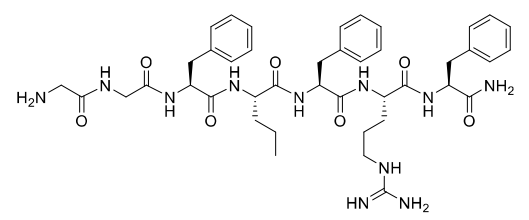

D

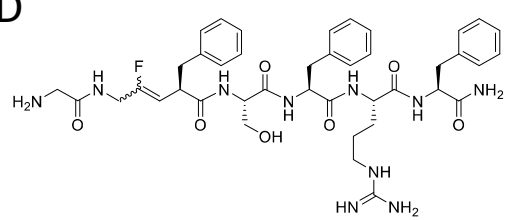

B

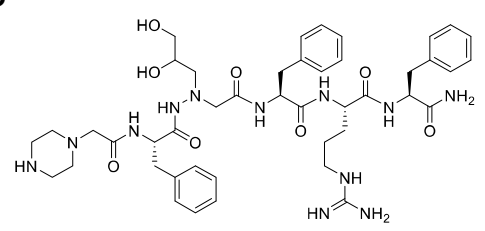

$E$

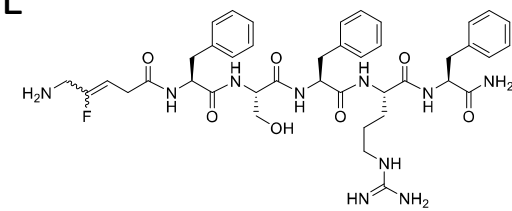

Figure 2. Chemical structures of peptidic and pseudopeptidic human QRFPR ligands. (A) $\left[\mathrm{Nva}^{23}\right] 26 \mathrm{RFa}(20-26)(\mathrm{LV}-2073)$; (B) $\left[\mathrm{Cmpi}^{21}{ }^{21}\right.$ aza- $\left.\beta^{3}-\mathrm{Hht}^{23}\right] 26 \mathrm{RFa}_{(21-26)}(\mathrm{LV}-2172) ;(\mathrm{C})\left[(\mathrm{Me})^{\omega}{ }^{\omega} \mathrm{Arg}^{25}\right] 26 \mathrm{RFa}_{(20-26)}(\mathrm{LV}-2186) ;(\mathrm{D})\left[\psi(\mathrm{CF}=\mathrm{CH})^{21,22}\right] 26 \mathrm{RFa}_{(20-26)}$;

(E) $\left[\psi(\mathrm{CF}=\mathrm{CH})^{20,21}\right] 26 \mathrm{RFa}(20-26)$.

Thus, the aim of the present study was to investigate molecular diversity at the unexplored Phe positions to go deeper into the structure-activity relationships of $26 \mathrm{RFa}$ for the design of potent $h$ QRFPR agonists.

\section{Results and Discussion}

\subsection{Impact of Modifications of Each Phenylalanine Residue}

It is well-accepted that the (hetero)aromatic units of peptides and proteins are master residues for DNA recognition [41], folding [42] and receptor-ligand interaction [43]. In particular, His, Phe, Trp and Tyr contribute to three types of non-covalent interactions including $\pi$-hydrogen bonds, electrostatic cation- $\pi$ interactions and van der Waals $\pi-\pi$ interactions [44] for governing molecular recognition of a ligand into the binding site of the receptor and transduction processes.

Replacement of all three phenylalanine residues of $26 \mathrm{RFa}_{(20-26)}(\mathrm{LV}-2021,1)$ with an alanine moiety identifies $\mathrm{Phe}^{24}$ and $\mathrm{Phe}^{26}$ as key residues for QRFPR activation, unlike $\mathrm{Phe}^{22}$ which is rather permissive to this substitution and thus weakly participates in the activity of the peptide [36]. Accordingly, $\mathrm{Phe}^{24}$ and $\mathrm{Phe}^{26}$ side chains strongly interact with hydrophobic regions at the bottom of the binding pocket of the $h$ QRFPR homology model [45]. In order to optimize positions 22, 24 and 26 of LV-2021, we have successively replaced each phenylalanine of the heptapeptide 1 with different commercially available aromatic or aliphatic building blocks (Table 1, compounds 2-48) and evalu-

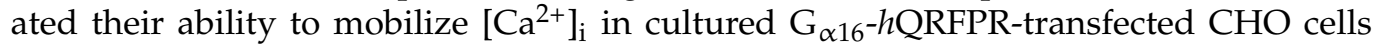
(Table 2), as previously described [36]. Substitution with the isosteric residue 3-(2-thienyl)alanine (Thi) yielded compounds LV-2050, LV-2051 and LV-2052 (2-4) that were less active than the parent peptide whatever the position concerned (Table 2). Similarly, incorporation of more hindered residues such as 3-(2-naphtyl)-alanine (2Nal; LV-2065, LV-2213, LV-2191, 5-7) and tryptophane (LV-2210, LV-2204, LV-2187, 8-10) altered the agonistic activity of the compounds, suggesting that there was little space available at the bottom of the orthosteric binding site as previously reported [45]. However, the para position of the $\mathrm{Phe}^{26}$ residue tolerated a bulky group or a substituent with different electronic effects. Indeed, $\left[p t \mathrm{BuPhe}^{26}{ }_{26 \mathrm{RFa}}(20-26)(\mathrm{LV}-2238,13),\left[\mathrm{Pcp}^{26}\right] 26 \mathrm{RFa}_{(20-26)}(\mathrm{LV}-2193,16)\right.$ and $\left[p \mathrm{NO}_{2} \mathrm{Phe}^{26}\right] 26 \mathrm{RFa}_{(20-26)}(\mathrm{LV}-2194,19)$ were significantly more potent than $26 \mathrm{RFa}(20-26)$ $(\mathrm{LV}-2021,1)$ to increase $\left[\mathrm{Ca}^{2+}\right]_{\mathrm{i}}$ in vitro (Table 2, Figure $\left.3 \mathrm{~A}-\mathrm{C}\right)$. The same substitutions at positions $\mathrm{Phe}^{22}$ and Phe ${ }^{24}$ were either neutral $(\mathbf{1 5}, \mathbf{1 7})$ or deleterious to activity $(\mathbf{1 1}, \mathbf{1 2}, \mathbf{1 4}, \mathbf{1 8})$. Finally, methylene shortening of the phenylalanine side chain led to the inactive phenylglycine (Phg)-containing $\left[\mathrm{Phg}^{22}\right] 26 \mathrm{RFa}_{(20-26)}(\mathrm{LV}-2053,20),\left[\mathrm{Phg}^{24}\right] 26 \mathrm{RFa}_{(20-26)}(\mathrm{LV}-2054,21$; LV-2055, 22) and $\left[\mathrm{Phg}^{26}\right] 26 \mathrm{RFa}_{(20-26)}(\mathrm{LV}-2056,23 ; \mathrm{LV}-2057,24)$ diastereoisomers, probably 
due to the lack of interactions with hydrophobic residues in the core of the $h \mathrm{QRFPR}$ binding pocket.

Table 1. Chemical data for $26 \mathrm{RFa}_{(20-26)}$ analogs substituted in positions 22,24 and 26.

\begin{tabular}{|c|c|c|c|c|c|c|c|}
\hline & \multirow{2}{*}{ Peptide } & \multirow{2}{*}{ Structure of Residue 22, 24, 26} & \multirow{2}{*}{ Code } & \multicolumn{2}{|c|}{ HPLC } & \multicolumn{2}{|c|}{ MS } \\
\hline & & & & $\operatorname{Rt}(\min )^{a}$ & Purity (\%) & Calcd $^{b}$ & Obsd $^{c}$ \\
\hline 1 & $26 \mathrm{RFa}_{(20-26)}$ & & LV-2021 & 18.0 & 99.9 & 815.41 & 816.53 \\
\hline 2 & {$\left[\mathrm{Thi}^{22}\right] 26 \mathrm{RFa}_{(20-26)}$} & & LV-2050 & 18.6 & 99.9 & 821.36 & 822.33 \\
\hline 3 & {$\left[\mathrm{Thi}^{24}\right] 26 \mathrm{RFa}_{(20-26)}$} & & LV-2051 & 18.6 & 99.9 & 821.36 & 822.37 \\
\hline 4 & {$\left[\mathrm{Thi}^{26}\right] 26 \mathrm{RFa}_{(20-26)}$} & & LV-2052 & 18.6 & 99.9 & 821.36 & 822.38 \\
\hline 5 & {$\left[2 \mathrm{Nal}^{22}\right] 26 \mathrm{RFa}_{(20-26)}$} & & LV-2065 & 20.4 & 99.9 & 865.42 & 866.45 \\
\hline 6 & {$\left[2 \mathrm{Nal}^{24}\right] 26 \mathrm{RFa}_{(20-26)}$} & & LV-2213 & 20.8 & 99.9 & 865.42 & 866.46 \\
\hline 7 & {$\left[2 \mathrm{Nal}^{26}\right] 26 \mathrm{RFa}_{(20-26)}$} & & LV-2191 & 20.6 & 99.9 & 865.42 & 866.57 \\
\hline 8 & {$\left[\operatorname{Trp}^{22}\right] 26 \mathrm{RFa}_{(20-26)}$} & & LV-2210 & 18.9 & 99.9 & 854.42 & 855.27 \\
\hline 9 & {$\left[\operatorname{Trp}^{24}\right] 26 \mathrm{RFa}_{(20-26)}$} & & LV-2204 & 18.6 & 99.9 & 854.42 & 855.43 \\
\hline 10 & {$\left[\operatorname{Trp}^{26}\right] 26 \mathrm{RFa}_{(20-26)}$} & & LV-2187 & 19.3 & 99.9 & 854.42 & 855.48 \\
\hline 11 & {$\left[p t\right.$ BuPhe $\left.^{22}\right] 26 R_{F} a_{(20-26)}$} & & LV-2068 & 22.1 & 99.9 & 871.47 & 872.49 \\
\hline 12 & {$\left[p t \mathrm{BuPhe}^{24}\right] 26 \mathrm{RFa}_{(20-26)}$} & & LV-2235 & 22.3 & 99.9 & 871.47 & 872.45 \\
\hline 13 & {$\left[p t \mathrm{BuPhe}^{26}\right] 26 \mathrm{RFa}_{(20-26)}$} & & LV-2238 & 22.4 & 99.9 & 871.47 & 872.55 \\
\hline 14 & {$\left[\mathrm{Pcp}^{22}\right] 26 \mathrm{RFa}_{(20-26)}$} & & LV-2069 & 19.9 & 99.9 & 849.37 & 850.30 \\
\hline 15 & {$\left[\mathrm{Pcp}^{24}\right] 26 \mathrm{RFa}_{(20-26)}$} & & LV-2232 & 20.5 & 99.9 & 849.37 & 850.41 \\
\hline 16 & {$\left[\mathrm{Pcp}^{26}\right] 26 \mathrm{RFa}_{(20-26)}$} & & LV-2193 & 20.0 & 99.9 & 849.37 & 850.42 \\
\hline 17 & {$\left[p \mathrm{NO}_{2} \mathrm{Phe}^{22}\right] 26 \mathrm{RFa}_{(20-26)}$} & & LV-2214 & 19.9 & 99.9 & 860.39 & 861.53 \\
\hline 18 & {$\left[p \mathrm{NO}_{2} \mathrm{Phe}^{24}\right] 26 \mathrm{RFa}_{(20-26)}$} & & LV-2236 & 19.0 & 99.9 & 860.39 & 861.46 \\
\hline 19 & {$\left[p \mathrm{NO}_{2} \mathrm{Phe}^{26}\right] 26 \mathrm{RFa}_{(20-26)}$} & & LV-2194 & 18.9 & 99.9 & 860.39 & 861.56 \\
\hline 20 & {$\left[\mathrm{Phg}^{22}\right] 26 \mathrm{RFa}_{(20-26)}$ dia 2} & & LV-2053 & 24.2 & 97.8 & 801.39 & 802.40 \\
\hline 21 & {$\left[\mathrm{Phg}^{24}\right] 26 \mathrm{RFa}_{(20-26)}$ dia 1} & & LV-2054 & 21.1 & 99.9 & 801.39 & 802.40 \\
\hline 22 & {$\left[\mathrm{Phg}^{24}\right] 26 \mathrm{RFa}_{(20-26)}$ dia 2} & & LV-2055 & 22.2 & 98.3 & 801.39 & 802.40 \\
\hline 23 & {$\left[\mathrm{Phg}^{26}\right] 26 \mathrm{RFa}_{(20-26)}$ dia 1} & & LV-2056 & 21.0 & 99.9 & 801.39 & 802.70 \\
\hline 24 & {$\left[\mathrm{Phg}^{26}\right] 26 \mathrm{RFa}_{(20-26)}$ dia 2} & & LV-2057 & 21.6 & 97.3 & 801.39 & 802.62 \\
\hline 25 & {$\left[\mathrm{NMePhe}^{22}\right] 26 \mathrm{RFa}_{(20-26)}$} & & LV-2066 & 18.9 & 99.9 & 829.42 & 830.53 \\
\hline 26 & {$\left[\mathrm{NMePhe}^{24}\right] 26 \mathrm{RFa}_{(20-26)}$} & & LV-2233 & 19.2 & 98.9 & 829.42 & 830.48 \\
\hline 27 & {$\left[\mathrm{NMePhe}^{26}\right] 26 \mathrm{RFa}_{(20-26)}$} & & LV-2242 & 18.8 & 99.9 & 829.42 & 830.48 \\
\hline 28 & {$\left[\mathrm{Tic}^{22}\right] 26 \mathrm{RFa}_{(20-26)}$} & & LV-2211 & 20.3 & 99.9 & 827.41 & 828.42 \\
\hline 29 & {$\left[\mathrm{Tic}^{24}\right] 26 \mathrm{RFa}_{(20-26)}$} & & LV-2205 & 17.7 & 99.9 & 827.41 & 828.46 \\
\hline 30 & {$\left[\mathrm{Tic}^{26}\right] 26 \mathrm{RFa}_{(20-26)}$} & & LV-2189 & 18.5 & 99.9 & 827.41 & 828.53 \\
\hline 31 & {$\left[N \mathrm{Nhe}^{22}\right] 26 \mathrm{RFa}_{(20-26)}$} & & LV-2058 & 21.1 & 99.9 & 815.41 & 816.23 \\
\hline 32 & {$\left[N \mathrm{Nhe}^{24}\right] 26 \mathrm{RFa}_{(20-26)}$} & & LV-2060 & 17.9 & 99.9 & 815.41 & 816.56 \\
\hline 33 & {$\left[N P h e^{26}\right] 26 R^{2} a_{(20-26)}$} & & LV-2061 & 18.6 & 99.9 & 815.41 & 816.40 \\
\hline 34 & {$\left[\mathrm{AHPPA}^{22}\right] 26 \mathrm{RFa}_{(20-26)}$} & & LV-2062 & 17.8 & 99.9 & 859.43 & 860.30 \\
\hline 35 & {$\left[\mathrm{AHPPA}^{24}\right] 26 \mathrm{RFa}_{(20-26)}$} & & LV-2063 & 17.7 & 99.9 & 859.43 & 860.40 \\
\hline 36 & {$\left[\mathrm{AHPPA}^{26}\right] 26 \mathrm{RFa}_{(20-26)}$} & & LV-2064 & 18.1 & 99.9 & 859.43 & 860.55 \\
\hline 37 & {$\left[\mathrm{DTrp}^{22}\right] 26 \mathrm{RFa}_{(20-26)}$} & & LV-2237 & 18.2 & 99.9 & 854.42 & 855.56 \\
\hline 38 & {$\left[\mathrm{DTr} \mathrm{T}^{24}\right] 26 \mathrm{RFa}_{(20-26)}$} & & LV-2070 & 17.7 & 99.9 & 854.42 & 855.53 \\
\hline 39 & {$\left[\mathrm{DTr} \mathrm{T}^{26}\right] 26 \mathrm{RFa}_{(20-26)}$} & & LV-2188 & 18.2 & 99.9 & 854.42 & 855.39 \\
\hline 40 & {$\left[\mathrm{DTic}^{22}\right] 26 \mathrm{RFa}_{(20-26)}$} & & LV-2212 & 18.4 & 99.9 & 827.41 & 828.49 \\
\hline 41 & {$\left[\mathrm{DTic}^{24}\right] 26 \mathrm{RFa}{ }_{(20-26)}$} & & LV-2215 & 18.2 & 99.9 & 827.41 & 828.36 \\
\hline 42 & {$\left[\mathrm{DTic}^{26}\right] 26 \mathrm{RFa}_{(20-26)}$} & & LV-2190 & 18.3 & 99.9 & 827.41 & 828.41 \\
\hline 43 & {$\left[\mathrm{Oic}^{22}\right] 26 \mathrm{RFa}_{(20-26)}$} & & LV-2067 & 18.6 & 99.9 & 819.44 & 820.56 \\
\hline 44 & {$\left[\mathrm{Oic}^{24}\right] 26 \mathrm{RFa}_{(20-26)}$} & & LV-2234 & 18.3 & 99.9 & 819.44 & 820.36 \\
\hline 45 & {$\left[\mathrm{Oic}^{26}\right] 26 \mathrm{RFa}_{(20-26)}$} & & LV-2241 & 18.1 & 99.9 & 819.44 & 820.45 \\
\hline 46 & {$\left[\mathrm{Cys}^{22,24}\right] 26 \mathrm{RFa}_{(20-26)}$} & & LV-2105 & 11.5 & 99.9 & 725.27 & 726.28 \\
\hline 47 & {$\left[\mathrm{Cys}^{22,26}\right] 26 \mathrm{RFa}_{(20-26)}$} & & LV-2107 & 6.7 & 99.9 & 725.27 & 726.37 \\
\hline 48 & {$\left[\mathrm{Cys}^{24,26}\right] 26 \mathrm{RFa}_{(20-26)}$} & & LV-2106 & 11.8 & 99.9 & 725.27 & 726.32 \\
\hline
\end{tabular}

${ }^{a}$ Retention time determined by analytical RP-HPLC. ${ }^{b}$ Theorical monoisotopic molecular weight. ${ }^{c} m / z[M H]^{+}$value assessed by MALDITOF-MS. AHPPA, (3S,4S)-4-amino-3-hydroxy-5-phenylpentanoic acid; 2Nal, 3-(2-naphtyl)-alanine; NMePhe, N-methyl-phenylalanine; NPhe, N-benzyl-glycine; Oic, octahydroindole-2-carboxylic acid; Pcp, (4-chloro)-phenylalanine; Phg, phenylglycine; $p \mathrm{NO}_{2} \mathrm{Phe}$, (4-nitro)phenylalanine; $p t$ BuPhe, (4-terbutyl)-phenylalanine; Thi, 3-(2-thienyl)-alanine; Tic, 1,2,3,4-tetrahydroisoquinoline-3-carboxylic acid. Diastereoisomers are numbered according to their elution order in RP-HPLC. 
Table 2. Biological data for $26 \mathrm{RFa}_{(20-26)}$ analogs substituted in positions 22,24 and 26.

\begin{tabular}{|c|c|c|c|c|c|c|c|c|c|}
\hline & \multirow{2}{*}{ Peptide } & \multirow{2}{*}{ Code } & \multicolumn{3}{|c|}{$\mathrm{EC}_{50}$} & \multicolumn{3}{|c|}{$\mathrm{IC}_{50}$} & Imax \\
\hline & & & \multicolumn{3}{|c|}{$(\mathrm{nM})$} & \multicolumn{3}{|c|}{$(\mathrm{nM})$} & $(\%)^{a}$ \\
\hline 1 & $26 \mathrm{RFa}_{(20-26)}$ & LV-2021 & 1640 & \pm & 259 & & - & & \\
\hline 2 & {$\left[\mathrm{Thi}^{22}\right] 26 \mathrm{RFa} \mathrm{a}_{(20-26)}$} & LV-2050 & 3160 & \pm & 1110 & & - & & \\
\hline 3 & {$\left[\mathrm{Thi}^{24}\right] 26 \mathrm{RFa}_{(20-26)}$} & LV-2051 & 2530 & \pm & 1020 & & - & & \\
\hline 4 & {$\left[\mathrm{Thi}^{26}\right] 26 \mathrm{RFa}_{(20-26)}$} & LV-2052 & 7227 & \pm & 96 & & - & & \\
\hline 5 & {$\left[2 \mathrm{Nal}^{22}\right] 26 \mathrm{RFa}_{(20-26)}$} & LV-2065 & 1150 & \pm & 170 & & - & & \\
\hline 6 & {$\left[2 \mathrm{Nal}^{24}\right] 26 \mathrm{RFa}_{(20-26)}$} & LV-2213 & 15,300 & \pm & 5400 & & - & & \\
\hline 7 & {$\left[2 \mathrm{Nal}^{26}{ }^{26}\right] 26 \mathrm{RFa}(20-26)$} & LV-2191 & 3790 & \pm & 1800 & & - & & \\
\hline 8 & {$\left[\operatorname{Trp}^{22}\right] 26 \mathrm{RFa}_{(20-26)}$} & LV-2210 & 1980 & \pm & 880 & & - & & \\
\hline 9 & {$\left[\operatorname{Trp}^{24}\right] 26 \mathrm{RFa}(20-26)$} & LV-2204 & 2040 & \pm & 1600 & & - & & \\
\hline 10 & {$\left[\operatorname{Trp}^{26}\right] 26 \mathrm{RFa}_{(20-26)}$} & LV-2187 & 5060 & \pm & 1300 & & - & & \\
\hline 11 & {$\left[p t \mathrm{BuPhe}^{22}\right] 26 \mathrm{RFa}_{(20-26)}$} & LV-2068 & & $>10,000$ & & 6220 & \pm & 2500 & 45 \\
\hline 12 & {$\left[p t \mathrm{BuPhe}^{24}\right] 26 \mathrm{RFa}_{(20-26)}$} & LV-2235 & & $>10,000$ & & & ND & & \\
\hline 13 & {$\left[p t \mathrm{BuPhe}^{26}\right] 26 \mathrm{RFa}_{(20-26)}$} & LV-2238 & 1040 & \pm & $25^{* *}$ & & - & & \\
\hline 14 & {$\left[\mathrm{Pcp}^{22}\right] 26 \mathrm{RFa}_{(20-26)}$} & LV-2069 & 5000 & \pm & 4500 & & - & & \\
\hline 15 & {$\left[\mathrm{Pcp}^{24}\right] 26 \mathrm{RFa}_{(20-26)}$} & LV-2232 & 850 & \pm & $240^{N S}$ & & - & & \\
\hline 16 & {$\left[\mathrm{Pcp}^{26}\right] 26 \mathrm{RFa}_{(20-26)}$} & LV-2193 & 457 & \pm & $71 * *$ & & - & & \\
\hline 17 & {$\left[p \mathrm{NO}_{2} \mathrm{Phe}^{22}\right] 26 \mathrm{RFa}_{(20-26)}$} & LV-2214 & 2130 & \pm & 1100 & & - & & \\
\hline 18 & {$\left[p \mathrm{NO}_{2} \mathrm{Phe}^{24}\right] 26 \mathrm{RFa}_{(20-26)}$} & LV-2236 & & $>10,000$ & & & ND & & \\
\hline 19 & {$\left[p \mathrm{NO}_{2} \mathrm{Phe}^{26}\right] 26 \mathrm{RFa}_{(20-26)}$} & LV-2194 & 491 & \pm & $33^{* *}$ & & - & & \\
\hline 20 & {$\left[\mathrm{Phg}^{22}\right] 26 \mathrm{RFa}_{(20-26)}$ dia 2} & LV-2053 & 5010 & \pm & 3600 & & - & & \\
\hline 21 & {$\left[\mathrm{Phg}^{24}\right] 26 \mathrm{RFa}_{(20-26)}$ dia 1} & LV-2054 & & $>10,000$ & & & ND & & \\
\hline 22 & {$\left[\mathrm{Phg}^{24}\right] 26 \mathrm{RFa}_{(20-26)}$ dia 2} & LV-2055 & & $>10,000$ & & & ND & & \\
\hline 23 & {$\left[\mathrm{Phg}^{26}\right] 26 \mathrm{RFa}_{(20-26)}$ dia 1} & LV-2056 & & $>10,000$ & & & ND & & \\
\hline 24 & {$\left[\mathrm{Phg}^{26}\right] 26 \mathrm{RFa}_{(20-26)}$ dia 2} & LV-2057 & & $>10,000$ & & & ND & & \\
\hline 25 & {$\left[N M e P h e^{22}\right] 26 R_{F a}(20-26)$} & LV-2066 & 15,100 & \pm & 4200 & & - & & \\
\hline 26 & {$\left[\mathrm{NMePhe}^{24}\right] 26 \mathrm{RFa}_{(20-26)}$} & LV-2233 & 1360 & \pm & 750 & & - & & \\
\hline 27 & {$\left[\mathrm{NMePhe}^{26}\right] 26 \mathrm{RFa}_{(20-26)}$} & LV-2242 & & $>10,000$ & & & ND & & \\
\hline 28 & {$\left[\mathrm{Tic}^{22}\right] 26 \mathrm{RFa}_{(20-26)}$} & LV-2211 & 327 & \pm & $170 *$ & & - & & \\
\hline 29 & {$\left[\mathrm{Tic}^{24}\right] 26 \mathrm{RFa}_{(20-26)}$} & LV-2205 & & $>10,000$ & & & ND & & \\
\hline 30 & {$\left[\mathrm{Tic}^{26}\right] 26 \mathrm{RFa}(20-26)$} & LV-2189 & 7700 & \pm & 4400 & & - & & \\
\hline 31 & {$\left[N P^{22}{ }^{26}{ }^{26 R a}(20-26)\right.$} & LV-2058 & 578 & \pm & $110 *$ & & - & & \\
\hline 32 & {$\left[N \mathrm{Phe}^{24}\right] 26 \mathrm{RFa}_{(20-26)}$} & LV-2060 & & $>10,000$ & & & ND & & \\
\hline 33 & {$\left[N P h e^{26}\right] 26 \mathrm{RFa}_{(20-26)}$} & LV-2061 & & $>10,000$ & & & ND & & \\
\hline 34 & {$\left[\mathrm{AHPPA}^{22}\right] 26 \mathrm{RFa}_{(20-26)}$} & LV-2062 & 2850 & \pm & 380 & & - & & \\
\hline 35 & [AHPPA $\left.^{24}\right] 26 \mathrm{RFa}_{(20-26)}$ & LV-2063 & & $>10,000$ & & & ND & & \\
\hline 36 & {$\left[\mathrm{AHPPA}^{26}\right] 26 \mathrm{RFa}_{(20-26)}$} & LV-2064 & & $>10,000$ & & & ND & & \\
\hline 37 & {$\left[\mathrm{DTrp}^{22}\right] 26 \mathrm{RFa}_{(20-26)}$} & LV-2237 & 6160 & \pm & 2400 & & - & & \\
\hline 38 & {$\left[\mathrm{DTrp}{ }^{24}\right] 26 \mathrm{RFa}_{(20-26)}$} & LV-2070 & & $>10,000$ & & & ND & & \\
\hline 39 & {$\left[\mathrm{DTr} \mathrm{T}^{26}\right] 26 \mathrm{RFa}_{(20-26)}$} & LV-2188 & & $>10,000$ & & & $>10,000$ & & 46 \\
\hline 40 & {$\left[\mathrm{DTic}^{22}\right] 26 \mathrm{RFa}_{(20-26)}$} & LV-2212 & 4990 & \pm & 2100 & & - & & \\
\hline 41 & {$\left[\mathrm{DTic}^{24}\right] 26 \mathrm{RFa}_{(20-26)}$} & LV-2215 & 6200 & \pm & 2600 & & - & & \\
\hline 42 & {$\left[\mathrm{DTic}^{26}\right] 26 \mathrm{RFa}_{(20-26)}$} & LV-2190 & & $>10,000$ & & & ND & & \\
\hline 43 & {$\left[\mathrm{Oic}^{22}\right] 26 \mathrm{RFa}_{(20-26)}$} & LV-2067 & & $>10,000$ & & & ND & & \\
\hline 44 & {$\left[\mathrm{Oic}^{24}\right] 26 \mathrm{RFa}_{(20-26)}$} & LV-2234 & & $>10,000$ & & & ND & & \\
\hline 45 & {$\left[\mathrm{Oic}^{26}\right] 26 \mathrm{RFa}_{(20-26)}$} & LV-2241 & & $>10,000$ & & & ND & & \\
\hline 46 & {$\left[\mathrm{Cys}^{22,24}\right] 26 \mathrm{RFa}_{(20-26)}$} & LV-2105 & & $>10,000$ & & & ND & & \\
\hline 47 & {$\left[\mathrm{Cys}^{22,26}\right] 26 \mathrm{RFa}_{(20-26)}$} & LV-2107 & & $>10,000$ & & & ND & & \\
\hline 48 & {$\left[\mathrm{Cys}^{24,26}\right] 26 \mathrm{RFa}_{(20-26)}$} & LV-2106 & & $>10,000$ & & & ND & & \\
\hline
\end{tabular}

${ }^{\text {a }}$ Inhibition at a maximal concentration of $10^{-5} \mathrm{M}$. Data are mean \pm SEM of at least three independent experiments performed in triplicate. AHPPA, (3S,4S)-4-amino-3-hydroxy-5-phenylpentanoic acid; 2Nal, 3-(2-naphtyl)-alanine; NMePhe, $N$-methyl-phenylalanine; $N$ Phe, N-benzyl-glycine; Oic, octahydroindole-2-carboxylic acid; Pcp, (4-chloro)-phenylalanine; Phg, phenylglycine; $p \mathrm{NO}_{2} \mathrm{Phe}$, (4-nitro)phenylalanine; $p t$ BuPhe, (4-terbutyl)-phenylalanine; Thi, 3-(2-thienyl)-alanine; Tic, 1,2,3,4-tetrahydroisoquinoline-3-carboxylic acid. Diastereoisomers are numbered according to their elution order in RP-HPLC. ND, not detectable. NS, not significant. ${ }^{*} p<0.05$, ${ }^{* *} p<0.01$ vs. control $26 \mathrm{RFa}_{(20-26)}(\mathrm{LV}-2021,1)$ as assessed by Mann and Whitney test. 

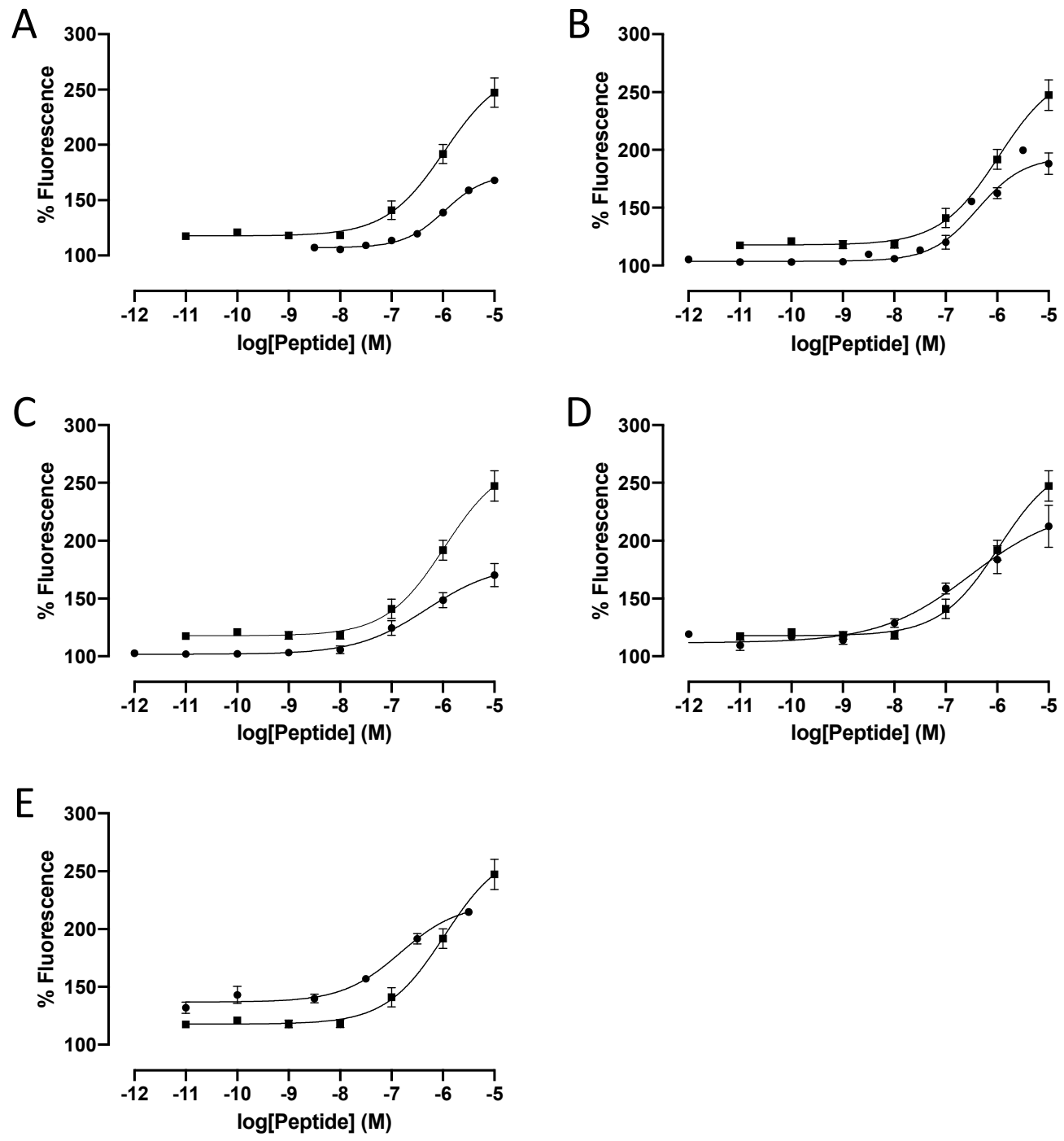

Figure 3. Effect of graded concentrations of Phe-modified $26 \mathrm{RFa}_{(20-26)}$ analogs on basal $\left[\mathrm{Ca}^{2+}\right]_{\mathrm{i}}$ mobilization in $\mathrm{G}_{\alpha 16}$ - $h \mathrm{QRFPR}$-transfected $\mathrm{CHO}$ cells. Prototype dose-response curves of $26 \mathrm{RFa}_{(20-26)}$ (LV-2021, 1, closed square, (A-E)) and its analogs (closed circles) [ $\left.p t \mathrm{BuPhe}^{26}\right] 26 \mathrm{RFa}(20-26)$ (LV2238, 13, (A)), [ $\left.\mathrm{Pcp}^{26}\right] 26 \mathrm{RFa}{ }_{(20-26)}(\mathrm{LV}-2193,16,(\mathbf{B})),\left[\mathrm{pNO}_{2} \mathrm{Phe}^{26}\right] 26 \mathrm{RFa}{ }_{(20-26)}(\mathrm{LV}-2194,19,(\mathbf{C}))$, $\left[\mathrm{Tic}^{22}\right] 26 \mathrm{RFa}_{(20-26)}(\mathrm{LV}-2211,28,(\mathbf{D}))$ and $\left[N \mathrm{Phe}^{22}\right] 26 \mathrm{RFa}_{(20-26)}(\mathrm{LV}-2058,31,(\mathbf{E}))$. Data are mean \pm SEM of triplicate. The $\mathrm{EC}_{50}$ calculated from these representative dose-response curves were $1069 \mathrm{nM}$ for 1 (LV-2021), $1076 \mathrm{nM}$ for 13 (LV-2238), $416 \mathrm{nM}$ for 16 (LV-2193), $489 \mathrm{nM}$ for 19 (LV-2194), $305 \mathrm{nM}$ for 28 (LV-2211) and $149 \mathrm{nM}$ for 31 (LV-2058).

The heptapeptide $26 \mathrm{RFa}_{(20-26)}$ does not encompass any secondary amide bonds within its backbone. Thus, nine analogs with $N$-methyl-phenylalanine (NMePhe), 1,2,3,4tetrahydroisoquinoline-3-carboxylic acid (Tic) or N-benzyl-glycine (NPhe) were synthesized. These modifications were aimed at analyzing the significance of H-bond contributions of the amide NHs. Alkylation of these NHs without loss of agonistic activity would facilitate further analog design. N-methylation of $\mathrm{Phe}^{22}(\mathrm{LV}-2066,25)$ and $\mathrm{Phe}^{26}(\mathrm{LV}-2242$, 27) nullified the activity of the parent compound $\left(\mathrm{EC}_{50}>10 \mu \mathrm{M}\right.$, Table 2$)$ revealing the critical role of the corresponding NHs in forming an H-bond with the $h$ QRFPR pocket residues or another residue of the peptide, whereas $N$-methylation of $\mathrm{Phe}^{24}$ (LV-2233, 26) did not impair the whole activity of LV-2021 (1). The corresponding Tic-containing analogs (28-30) did not totally confirm these data since $\left[\mathrm{Tic}^{24}\right] 26 \mathrm{RFa}_{(20-26)}(\mathrm{LV}-2205,29)$ and $\left[\mathrm{Tic}^{26}\right] 26 \mathrm{RFa}_{(20-26)}(\mathrm{LV}-2189,30)$ were devoid of effect while $\left[\mathrm{Tic}^{22}\right] 26 \mathrm{RFa}{ }_{(20-26)}(\mathrm{LV}-$ $2211,28)$ was 5 -fold more potent than $26 \mathrm{RFa}_{(20-26)}(\mathrm{LV}-2021,1)($ Figure 3D). It is thus also 
plausible that the structural constraint induced by the $\operatorname{Tic}^{22}$ residue may keep the side chain in a favorable conformation for receptor activation, which was less accessible by free rotation in the native Phe side chain. The NPhe-peptoid analogs (31-33) behaved like the Tic-containing counterparts, and $\left[\mathrm{NPhe}^{22}\right] 26 \mathrm{RFa}_{(20-26)}(\mathrm{LV}-2058,31)$ was almost as potent as LV-2211 (Table 2, Figure 3E). At this stage, conclusions are compromised since the local conformational rigidity to peptide backbone via pipecolinic acid bridge [46] and the shift of side chain functionality from the $\alpha$-carbon to the $\mathrm{NH}$ offering flexibility to peptoid led to similar results. However, we can speculate that the $\mathrm{Phe}^{22}$ residue, which does not dive deeply into the binding pocket, accommodated rather well with backbone modifications at the odds to Phe ${ }^{24}$ and $\mathrm{Phe}^{26}$ which are strongly embedded in the orthosteric binding site [45]. An increase in H-bond capacity and flexibility by the introduction of $(3 S, 4 S)$ 4-amino-3-hydroxy-5-phenylpentanoic acid (AHPPA), a marine Cyanobacterium symploca peptide naturally occurring $\gamma$-amino acid [47] (34-36) had no positive effect on the potency of the parent heptapeptide (Table 2).

We have previously reported that stereoinversion to D-Phe in $\left[\mathrm{DPhe}^{22}\right] 26 \mathrm{RFa} \mathrm{a}_{(20-26)}$, $\left[\mathrm{DPhe}^{24}\right] 26 \mathrm{RFa}_{(20-26)}$ and $\left[\mathrm{DPhe}{ }^{26}\right]_{26 \mathrm{RFa}_{(20-26)}}$ results in a complete loss of the $\left[\mathrm{Ca}^{2+}\right]_{\mathrm{i}}$ response [36]. Similarly, successive D-Trp and D-Tic incorporation within the $26 \mathrm{RFa}_{(20-26)}$ sequence in the same positions generated weak (LV-2237, 37; LV-2212, 40; LV-2215, 41) or inactive (LV-2070, 38; LV-2188, 39; LV-2190, 42) agonists (Table 2), confirming that not only side chain functionality but also its correct orientation play a critical role in the activity of the peptide.

Systematic replacement of the three Phe units with the constrained aliphatic residue octahydroindole-2-carboxylic acid (Oic) yielded LV-2067 (43), LV-2234 (44) and LV-2241 (45) that were totally devoid of $\mathrm{Ca}^{2+}$-mobilizing activity (Table 2). The Oic moiety might induce a turn, like the prolyl residue [48], that would destabilize at this point the correct folding of the peptide backbone in the $h$ QRFPR binding pocket. Furthermore, these results also confirm the importance of aromaticity in positions 22,24 and 26 of $26 \mathrm{RFa}_{(20-26)}$ in the receptor activation process.

In methanol, 26RFa adopts a well-defined conformation consisting of a N-terminal amphipathic $\alpha$-helical structure ( $\left.\mathrm{Pro}^{4}-\mathrm{Arg}^{17}\right)$, preceding a C-terminal disordered region [49]. Interestingly, it has been shown that two Phe residues in an $i, i+4$ arrangement ( $\mathrm{Phe}^{22}$ and $\mathrm{Phe}^{26}$ of $26 \mathrm{RFa}$ and $\left.26 \mathrm{RFa}_{(20-26)}\right)$ enthalpically stabilize an $\alpha$-helix [50]. To assess such a conformation of the C-terminal extremity of the peptide inside the $h$ QRFPR binding pocket during the recognition process, we prepared cyclic disulfide-bridged $26 \mathrm{RFa}_{(20-26)}$ analogs (Table 1, 46-48) as already reported for the pentapeptide Met-enkephalin [51]. The activity profiles of $\left[\mathrm{Cys}^{22,26}\right] 26 \mathrm{RFa}_{(20-26)}(\mathrm{LV}-2107,47),\left[\mathrm{Cys}^{22,24}\right] 26 \mathrm{RFa}_{(20-26)}(\mathrm{LV}-2105,46)$ and $\left[\mathrm{Cys}^{24,26}\right] 26 \mathrm{RFa}_{(20-26)}(\mathrm{LV}-2106,48)$ did not confirm that the Phe residues of $26 \mathrm{RFa}_{(20-26)}$ (LV-2021, 1) participate in the bioactive conformation of the peptide or that some mandatory features are absent of these analogs (Table 2).

Compounds inactive as agonists were evaluated as possible antagonists of $26 \mathrm{RFa}-$ evoked calcium increase in cultured $\mathrm{G}_{\alpha 16}-h \mathrm{QRFPR}$-transfected $\mathrm{CHO}$ cells. None of the members of this series were able to reverse the stimulatory effect of $10^{-7} \mathrm{M} 26 \mathrm{RFa}$ on $\left[\mathrm{Ca}^{2+}\right]_{\mathrm{i}}$, except LV-2068 (11) and LV-2188 (39) which antagonized $45-46 \%$ of the agonistic response with, respectively, modest and very low $\mathrm{IC}_{50}$ (Table 2, Figure 4).

To summarize, the three phenylalanine units exhibit differential contributions to the biological activity of $26 \mathrm{RFa}_{(20-26)}$. The Phe ${ }^{22}$ residue appears to be the most permissive as it tolerated $N$-substitution like in $\left[\mathrm{Tic}^{22}\right] 26 \mathrm{RFa}_{(20-26)}(\mathrm{LV}-2211,28)$ which was 5-folds more potent than the lead peptide. Conversely, most modifications of $\mathrm{Phe}^{24}$ and $\mathrm{Phe}^{26} \mathrm{did}$ not improve the activity with the exception of para substituents of lesser size than that of a tertbutyl group. Indeed, $\left[\mathrm{Pcp}^{24}\right] 26 \mathrm{RFa}{ }_{(20-26)}(\mathrm{LV}-2232,15),\left[\mathrm{Pcp}^{26}\right] 26 \mathrm{RFa}_{(20-26)}(\mathrm{LV}-2193$, 16) and $\left[\mathrm{pNO}_{2} \mathrm{Phe}^{26}\right] 26 \mathrm{RFa}_{(20-26)}(\mathrm{LV}-2194,19)$ were $2-, 3.5$ - and 3.3 -fold more potent than $26 \mathrm{RFa}_{(20-26)}(\mathrm{LV}-2021,1)$. Although the amide $\mathrm{NH}$ of $\mathrm{Phe}^{24}$ residue was probably not involved in $\mathrm{H}$-bond, our results suggest that some flexibility of the peptide backbone is required at this point. Noteworthy, not only the H-bond capacity, but other features were 
also impacted by these modifications such as the side chain presentation geometry, amide cis/trans isomerization equilibrium, and/or $\beta$-sheet potential of the analog with a wide range of steric, electronic and hydrophobic characteristics.

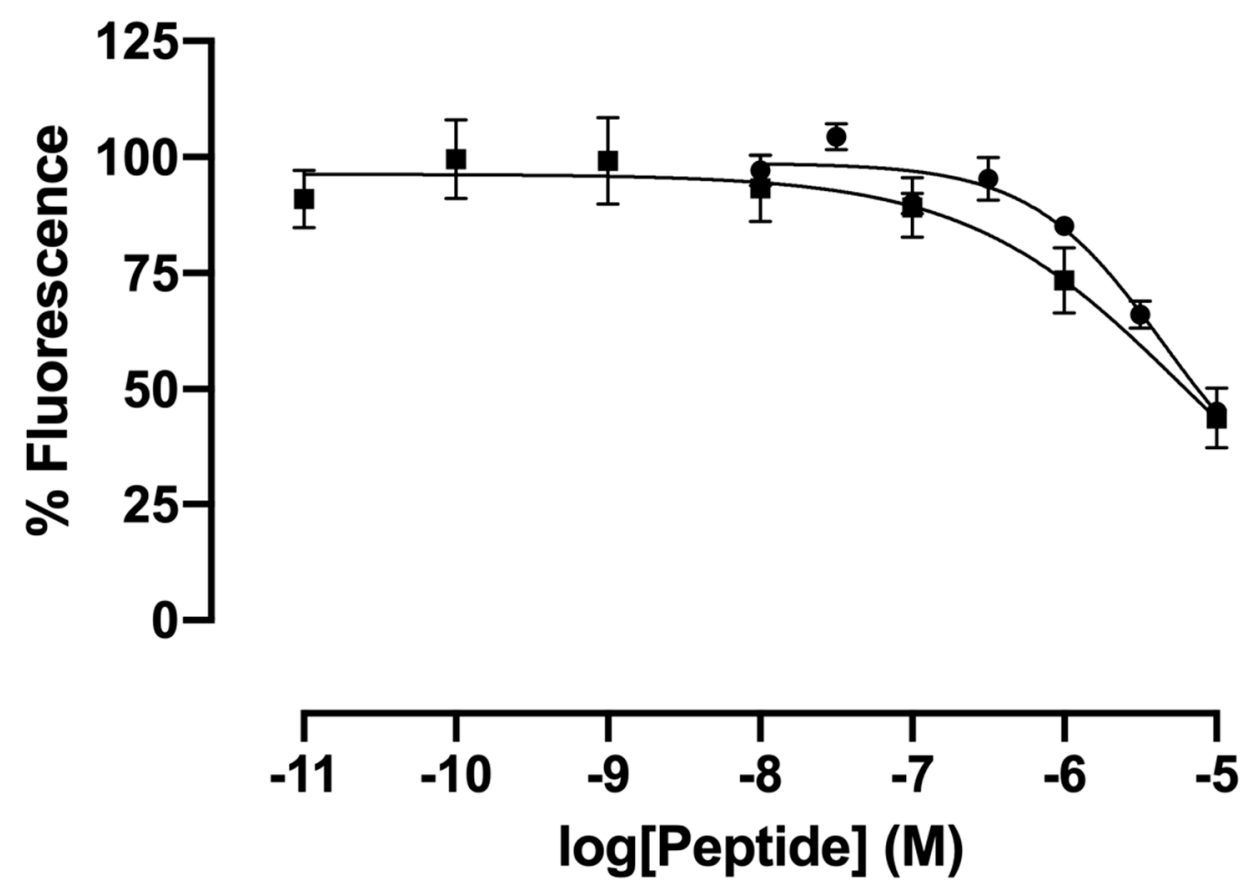

Figure 4. Effect of graded concentrations of Phe-modified $26 \mathrm{RFa}_{(20-26)}$ analogs on 26RFa-evoked $\left[\mathrm{Ca}^{2+}\right]_{i}$ mobilization in $\mathrm{G}_{\alpha 16}$-hQRFPR-transfected $\mathrm{CHO}$ cells. Prototype dose-inhibition curves of $\left[p t\right.$ BuPhe $\left.^{22}\right] 26 \mathrm{RFa}_{(20-26)}\left(\mathrm{LV}-2068,11\right.$, closed circles) and $\left[\mathrm{DTrp}^{26}\right] 26 \mathrm{RFa}{ }_{(20-26)}(\mathrm{LV}-2188,39$, closed squares) on $10^{-7} \mathrm{M} 26 \mathrm{RFa}$-evoked response. Data are mean $\pm \mathrm{SEM}$ of triplicate. The $\mathrm{IC}_{50}$ value calculated from these representative dose-inhibition curves were $4495 \mathrm{nM}$ for 11 (LV-2068) and 5841 $\mathrm{nM}$ for 39 (LV-2188).

\subsection{Impact of Concomitant Modifications of $\mathrm{Gly}^{20}, \mathrm{Gly}^{21}$ and Phe $\mathrm{P}^{22}$ Residues}

The variety of conformations characterized in enkephalins [52] and other short peptide models containing contiguous Gly units [53] reveals an important heterogeneity in 3D structures of the Gly-Gly segment influenced by both neighboring residues and environment. In the homology model of $h \mathrm{QRFPR}$, the Gly-Gly motif of $26 \mathrm{RFa}_{(19-26)}$ seems to adopt an extended conformation [45] generally observed in $\beta$-strands that form part of $\beta$-sheets. We have previously investigated the local requirement of the $\mathrm{N}$-terminal dipeptide extremity of $26 \mathrm{RFa}_{(20-26)}$ and one of the most interesting results has been achieved by introducing a 4-carboxymethylpiperazine (Cmpi) unit in place of the native Gly-Gly pair which leads to the 5-fold more potent analog $\left[\mathrm{Cmpi}^{21}\right] 26 \mathrm{RFa}{ }_{(21-26)}(\mathrm{LV}-2043)$ than the reference heptapeptide [37]. Herein, to probe the space around this peptidomimetic moiety, we have introduced 2-piperazino-2-aryl-acetic acid racemate derivatives (Figure 5) in place of the first three $26 \mathrm{RFa}_{(20-26)}$ residues (Table 3 ) and evaluated the in vitro activity of each diastereoisomer (Table 4). In this series, analogs LV-2102 (52) and LV-2175 (55) containing 2-piperazino-2-[(4-fluoro)phenyl]acetic acid (Cmpi(4-FPhg)) and 2-piperazino2 -[(2-fluoro)phenyl]acetic acid (Cmpi(2-FPhg)) respectively, demonstrated similar $\left[\mathrm{Ca}^{2+}\right]_{\mathrm{i}}{ }^{-}$ mobilizing activity in $h$ QRFPR-transfected cells compared to the lead peptide, while other derivatives exhibited slightly (LV-2101, 51; LV-2103, 53; LV-2104 54; LV-2183, 63) or detrimentally lower potency (Table 4). Altogether, these results suggest that incorporation of an aryl substituent on the methylene of the Cmpi building block with the concomitant deletion of the $\mathrm{Phe}^{22}$ residues do neither improve the ability of $\left[\mathrm{Cmpi}^{21}\right] 26 \mathrm{RFa}_{(21-26)}$ to activate $h \mathrm{QRFPR}$ nor reverse the 26RFa-evoked effect. 


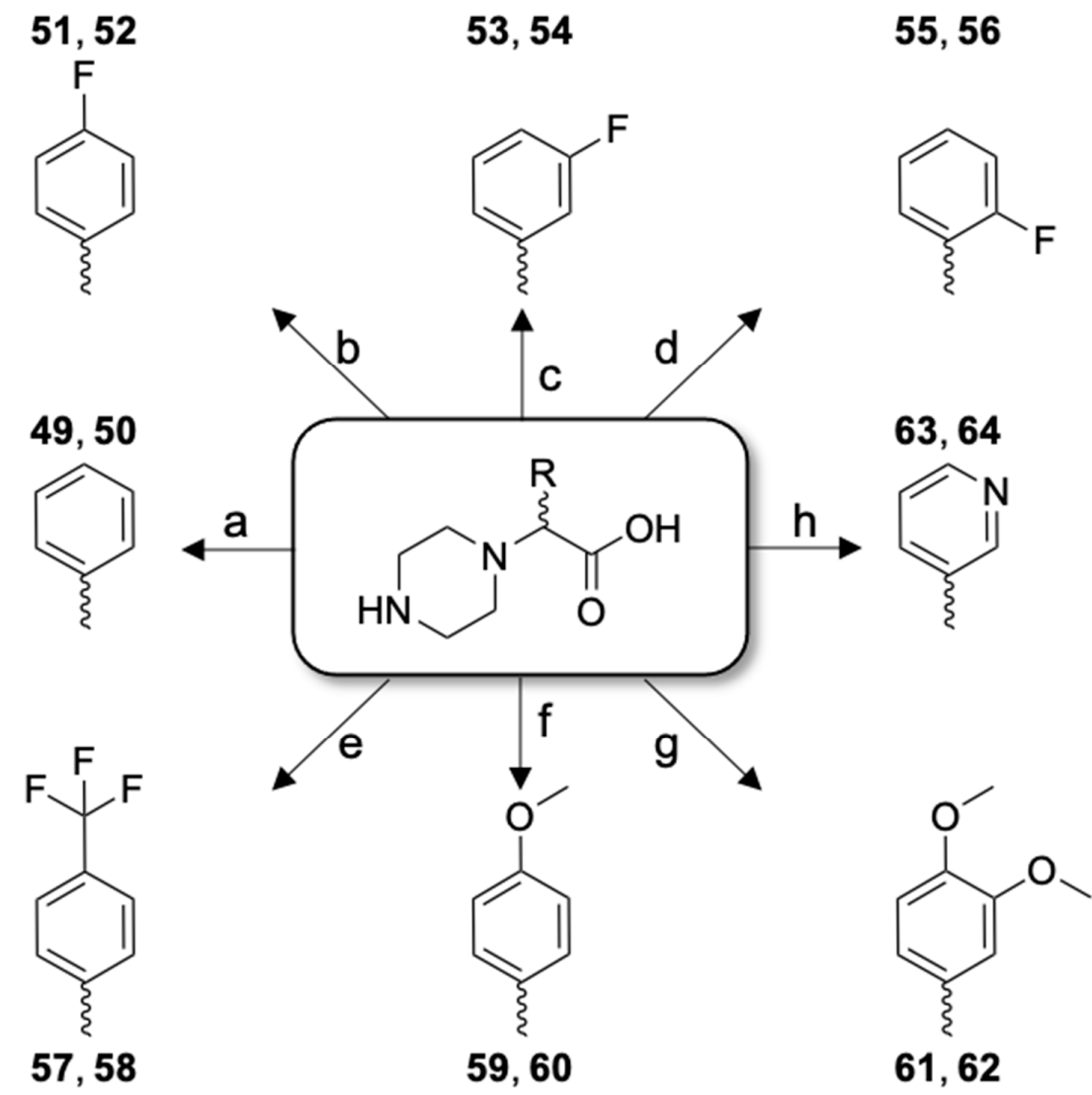

Figure 5. 2-Piperazino-2-aryl-acetic acid derivatives used in place of the H-Gly-Gly-Phe- sequence and incorporated in 49-64. These peptidomimetic moieties were used as racemate.

Table 3. Chemical data for $26 \mathrm{RFa}_{(22-26)}$ analogs.

\begin{tabular}{|c|c|c|c|c|c|c|}
\hline & \multirow{2}{*}{ Peptide Derivative } & \multirow{2}{*}{ Code } & \multicolumn{2}{|c|}{ HPLC } & \multicolumn{2}{|c|}{ MS } \\
\hline & & & $\operatorname{Rt}(\min )^{a}$ & Purity (\%) & Calcd $^{b}$ & Obsd $^{c}$ \\
\hline 1 & $26 \mathrm{RFa}_{(20-26)}$ & LV-2021 & 18.0 & 99.9 & 815.41 & 816.53 \\
\hline 49 & {$\left[\mathrm{Cmpi}(\mathrm{Phg})^{22}\right] 26 \mathrm{RFa}_{(22-26)}$ dia 1} & LV-2099 & 12.8 & 99.9 & 756.41 & 757.46 \\
\hline 50 & {$\left[\mathrm{Cmpi}(\mathrm{Phg})^{22}\right] 26 \mathrm{RFa}_{(22-26)}$ dia 2} & LV-2100 & 12.9 & 99.9 & 756.41 & 757.46 \\
\hline 51 & {$\left[\mathrm{Cmpi}(4-F P h g)^{22}\right] 26 \mathrm{RFa}_{(22-26)}$ dia 1} & LV-2101 & 15.3 & 99.9 & 774.40 & 775.37 \\
\hline 52 & {$\left[\mathrm{Cmpi}(4-\mathrm{FPhg})^{22}\right] 26 \mathrm{RFa}_{(22-26)}$ dia 2} & LV-2102 & 15.7 & 99.9 & 774.40 & 775.39 \\
\hline 53 & {$\left[\mathrm{Cmpi}(3-\mathrm{FPhg})^{22}\right] 26 \mathrm{RFa}_{(22-26)}$ dia 1} & LV-2103 & 15.3 & 99.9 & 774.40 & 775.42 \\
\hline 54 & {$\left[\mathrm{Cmpi}(3-\mathrm{FPhg})^{22}\right] 26 \mathrm{RFa}_{(22-26)}$ dia 2} & LV-2104 & 15.8 & 99.9 & 774.40 & 775.41 \\
\hline 55 & {$\left[\mathrm{Cmpi}(2-\mathrm{FPhg})^{22}\right] 26 \mathrm{RFa}_{(22-26)}$ dia 1} & LV-2175 & 13.9 & 98.4 & 774.40 & 775.47 \\
\hline 56 & {$\left[\mathrm{Cmpi}(2-\mathrm{FPhg})^{22}\right] 26 \mathrm{RFa}_{(22-26)}$ dia 2} & LV-2176 & 14.7 & 99.9 & 774.40 & 775.53 \\
\hline 57 & {$\left[\mathrm{Cmpi}(4-\mathrm{TfmPhg})^{22}\right] 26 \mathrm{RFa}_{(22-26)}$ dia 1} & LV-2177 & 25.3 & 99.9 & 824.39 & 825.47 \\
\hline 58 & {$\left[\mathrm{Cmpi}(4-\mathrm{TfmPhg})^{22}\right] 26 \mathrm{RFa}_{(22-26)}$ dia 2} & LV-2178 & 26.2 & 99.9 & 824.39 & 825.39 \\
\hline 59 & {$\left[\mathrm{Cmpi}(4-\mathrm{MeOPhg})^{22}\right] 26 \mathrm{RFa}_{(22-26)}$ dia 1} & LV-2179 & 13.2 & 99.9 & 786.42 & 787.47 \\
\hline 60 & {$\left[\mathrm{Cmpi}(4-\mathrm{MeOPhg})^{22}\right] 26 \mathrm{RFa}_{(22-26)}$ dia 2} & LV-2180 & 13.4 & 99.9 & 786.42 & 787.41 \\
\hline 61 & {$\left[\mathrm{Cmpi}(3,4 \text {-diMeOPhg })^{22}\right] 26 \mathrm{RFa}_{(22-26)}$ dia 1} & LV-2181 & 10.9 & 99.9 & 816.43 & 817.37 \\
\hline 62 & {$\left[\mathrm{Cmpi}(3,4 \text {-diMeOPhg })^{22}\right] 26 \mathrm{RFa}_{(22-26)}$ dia 2} & LV-2182 & 11.5 & 99.9 & 816.43 & 817.49 \\
\hline 63 & {$\left[\mathrm{Cmpi}(3-\mathrm{Pyg})^{22}\right] 26 \mathrm{RFa}_{(22-26)}$ dia 1} & LV-2183 & 19.5 & 99.9 & 757.40 & 758.40 \\
\hline 64 & {$\left[\mathrm{Cmpi}(3-\mathrm{Pyg})^{22}\right] 26 \mathrm{RFa}_{(22-26)}$ dia 2} & LV-2184 & 20.3 & 99.9 & 757.40 & 758.31 \\
\hline
\end{tabular}

${ }^{a}$ Retention time determined by analytical RP-HPLC. ${ }^{b}$ Theorical monoisotopic molecular weight. ${ }^{c} \mathrm{~m} / z$ [MH] ${ }^{+}$value assessed by MALDI-TOF-MS. Cmpi(Phg), 2-piperazino-2-phenylacetic acid; Cmpi(2-FPhg), 2-piperazino-2-[(2-fluoro)phenyl]acetic acid; Cmpi(3-FPhg), 2-piperazino-2-[(3-fluoro)phenyl]acetic acid; Cmpi(4-FPhg), 2-piperazino-2-[(4-fluoro)phenyl]acetic acid; Cmpi(4-TfmPhg), 2-piperazino2-[(4-trifluoromethyl)phenyl]acetic acid; Cmpi(4-MeOPhg), 2-piperazino-2-[(4-methoxy)phenyl]acetic acid; Cmpi(3,4-diMeOPhg), 2piperazino-2-[(3,4-dimethoxy)phenyl]acetic acid; Cmpi(3-Pyg), 2-piperazino-2-(3-pyridyl)acetic acid. Diastereoisomers are numbered according to their elution order in RP-HPLC. 
Table 4. Biological data for $26 \mathrm{RFa}_{(22-26)}$ analogs.

\begin{tabular}{|c|c|c|c|c|c|c|}
\hline & \multirow{2}{*}{ Peptide Derivative } & \multirow{2}{*}{ Code } & \multicolumn{3}{|c|}{$\mathrm{EC}_{50}$} & \multirow{2}{*}{$\frac{\mathrm{IC}_{50}}{(\mathrm{nM})}$} \\
\hline & & & & $(\mathrm{nM})$ & & \\
\hline 1 & $26 \mathrm{RFa}_{(20-26)}$ & LV-2021 & 1640 & \pm & 259 & - \\
\hline 49 & {$\left[\mathrm{Cmpi}(\mathrm{Phg})^{22}\right] 26 \mathrm{RFa}_{(22-26)}$ dia 1} & LV-2099 & & $>10,000$ & & ND \\
\hline 50 & {$\left[\mathrm{Cmpi}(\mathrm{Phg})^{22}\right] 26 \mathrm{RFa}_{(22-26)}$ dia 2} & LV-2100 & & $>10,000$ & & ND \\
\hline 51 & {$\left[\mathrm{Cmpi}(4-\mathrm{FPhg})^{22}\right] 26 \mathrm{RFa}_{(22-26)}$ dia 1} & LV-2101 & 4050 & \pm & 1200 & - \\
\hline 52 & {$\left[\mathrm{Cmpi}(4-\mathrm{FPhg})^{22}\right] 26 \mathrm{RFa}_{(22-26)}$ dia 2} & LV-2102 & 1660 & \pm & 788 & - \\
\hline 53 & {$\left[\mathrm{Cmpi}(3-\mathrm{FPhg})^{22}\right] 26 \mathrm{RFa}_{(22-26)}$ dia 1} & LV-2103 & 3560 & \pm & 440 & - \\
\hline 54 & {$\left[\mathrm{Cmpi}(3-\mathrm{FPhg})^{22}\right] 26 \mathrm{RFa}_{(22-26)}$ dia 2} & LV-2104 & 2710 & \pm & 1000 & - \\
\hline 55 & {$\left[\mathrm{Cmpi}(2-\mathrm{FPhg})^{22}\right] 26 \mathrm{RFa}_{(22-26)}$ dia 1} & LV-2175 & 2480 & \pm & 1113 & - \\
\hline 56 & {$\left[\mathrm{Cmpi}(2-\mathrm{FPhg})^{22}\right] 26 \mathrm{RFa}_{(22-26)}$ dia 2} & LV-2176 & & $>10,000$ & & ND \\
\hline 57 & {$\left[\mathrm{Cmpi}(4-\mathrm{TfmPhg})^{22}\right] 26 \mathrm{RFa}_{(22-26)}$ dia 1} & LV-2177 & & $>10,000$ & & ND \\
\hline 58 & {$\left[\mathrm{Cmpi}(4-\mathrm{TfmPhg})^{22}\right] 26 \mathrm{RFa}_{(22-26)}$ dia 2} & LV-2178 & & $>10,000$ & & ND \\
\hline 59 & {$\left[\mathrm{Cmpi}(4-\mathrm{MeOPhg})^{22}\right] 26 \mathrm{RFa}_{(22-26)}$ dia 1} & LV-2179 & & $>10,000$ & & ND \\
\hline 60 & {$\left[\mathrm{Cmpi}(4-\mathrm{MeOPhg})^{22}\right] 26 \mathrm{RFa}_{(22-26)}$ dia 2} & LV-2180 & & $>10,000$ & & ND \\
\hline 61 & {$\left[\mathrm{Cmpi}(3,4-\mathrm{diMeOPhg})^{22}\right] 26 \mathrm{RFa}_{(22-26)}$ dia 1} & LV-2181 & & $>10,000$ & & ND \\
\hline 62 & {$\left[\mathrm{Cmpi}(3,4-\text { diMeOPhg })^{22}\right] 26 \mathrm{RFa}_{(22-26)}$ dia 2} & LV-2182 & & $>10,000$ & & ND \\
\hline 63 & {$\left[\mathrm{Cmpi}(3-\mathrm{Pyg})^{22}\right] 26 \mathrm{RFa}_{(22-26)}$ dia 1} & LV-2183 & 4990 & \pm & 2800 & - \\
\hline 64 & {$\left[\mathrm{Cmpi}(3-\mathrm{Pyg})^{22}\right] 26 \mathrm{RFa}_{(22-26)}$ dia 2} & LV-2184 & & $>10,000$ & & ND \\
\hline
\end{tabular}

Data are mean \pm SEM of at least three independent experiments performed in triplicate. Cmpi(Phg), 2-piperazino-2-phenylacetic acid; Cmpi(2-FPhg), 2-piperazino-2-[(2-fluoro)phenyl]acetic acid; Cmpi(3-FPhg), 2-piperazino-2-[(3-fluoro)phenyl]acetic acid; Cmpi(4-FPhg), 2-piperazino-2-[(4-fluoro)phenyl]acetic acid; Cmpi(4-TfmPhg), 2-piperazino-2-[(4-trifluoromethyl)phenyl]acetic acid; Cmpi(4-MeOPhg), 2-piperazino-2-[(4-methoxy)phenyl]acetic acid; Cmpi(3,4-diMeOPhg), 2-piperazino-2-[(3,4-dimethoxy)phenyl]acetic acid; Cmpi(3-Pyg), 2-piperazino-2-(3-pyridyl)acetic acid. Diastereoisomers are numbered according to their elution order in RP-HPLC. ND, not detectable.

\section{Materials and Methods}

\subsection{Reagents}

All Fmoc-amino acid residues and building blocks and O-benzotriazol-1-yl- $N, N, N^{\prime}, N^{\prime}-$ tetramethyluronium hexafluorophosphate (HBTU) were purchased from Christof Senn Laboratories (Dielsdorf, Switzerland) or PolyPeptide (Strasbourg, France). Rink amide 4-methylbenzhydrylamine (MBHA) resin was from Novabiochem (Darmstadt, Germany) and $N$-methylpyrrolidone (NMP), dimethylformamide (DMF) and dichloromethane (DCM) were from Biosolve (Dieuze, France). N,N-Diisopropylethylamine (DIEA), piperidine, trifluoroacetic acid (TFA), triisopropylsilane (TIS), tert-butylmethylether (TBME) were supplied from Sigma-Aldrich (Saint-Quentin-Fallavier, France). Acetonitrile was from Fisher Scientific (Illkirch, France) and $\alpha$-cyano-4-hydroxycinnamic acid (CHCA) matrix from LaserBioLabs (Valbonne, France).

\subsection{Peptide Synthesis and Purification}

All peptides and derivatives were synthesized by the solid phase methodology on Rink amide MBHA resin using a Liberty microwave-assisted automated peptide synthesizer (CEM, Saclay, France) and the standard manufacturer's procedures at $0.1 \mathrm{mmol}$ scale as previously described [54]. All Fmoc-amino acids and building blocks (0.5 mmol, 5 equiv) were coupled by in situ activation with HBTU ( $0.5 \mathrm{mmol}, 5$ equiv) and DIEA ( $1 \mathrm{mmol}$, 10 equiv). Peptides and derivatives were deprotected and cleaved from the resin by adding a TFA/TIS $/ \mathrm{H}_{2} \mathrm{O}(99.5 / 0.25 / 0.25)$ mixture for $120 \mathrm{~min}$ at room temperature. After filtration, crude peptides were precipitated by addition of TBME, centrifuged and recovered by elimination of the supernatant ( 3 folds). Peptides and derivatives were purified by reversedphase HPLC (RP-HPLC) on a $2.2 \times 25 \mathrm{~cm}$ Vydac $218 \mathrm{TP} 1022 \mathrm{C}_{18}$ column (Grace, Epernon, France) using a linear gradient $(10-40 \%, 10-50 \%, 10-60 \%, 20-40 \%, 20-50 \%$ or $20-60 \%$ over $45 \mathrm{~min})$ of acetonitrile/TFA $(99.9 / 0.1)$ at a flow rate of $10 \mathrm{~mL} / \mathrm{min}$. The purified peptides were then characterized by MALDI-TOF mass spectrometry on a UltrafleXtreme (Bruker, Strasbourg, France) using CHCA as a matrix. Analytical RP-HPLC, performed on a $0.46 \times 25 \mathrm{~cm}$ Vydac $218 \mathrm{TP} 54 \mathrm{C}_{18}$ column (Grace), showed that the purity of all compounds was $>97.3 \%$. 


\subsection{Cell Culture}

Stably transfected $h$ QRFPR CHO cells were obtained as previously described [36-38]. The cells were maintained in F-12 nutrient mixture (Ham-F12) medium supplemented with $10 \%$ fetal bovine serum, $2 \mathrm{mM}$ glutamine, and penicillin-streptomycin. Expression of $\mathrm{G}_{\alpha 16}$ cells was maintained using selection antibiotic hygromycin $B(200 \mu \mathrm{g} / \mathrm{mL})$ and that of $h$ QRFPR using geneticin G418 $(500 \mu \mathrm{g} / \mathrm{mL})$ (Life Technologies, Villebon-Sur-Yvette, France) in a humidified $5 \% \mathrm{CO}_{2}$ atmosphere at $37^{\circ} \mathrm{C}$.

\subsection{Calcium Mobilization Assays}

Changes in intracellular $\mathrm{Ca}^{2+}$ concentrations induced by $26 \mathrm{RFa}_{(20-26)}$ analogs in $\mathrm{CHO}-$ $\mathrm{G}_{\alpha 16}$ - $h \mathrm{QRFPR}$-transfected cells were measured on a benchtop scanning fluorometer Flexstation III (Molecular Devices, Sunnyvale, CA, USA) as previously described [36-38,55,56]. Briefly, 96-well assay black plates with clear bottoms (Corning international, Avon, France) were seeded at a density of 40,000 cells/well $24 \mathrm{~h}$ prior to assay. For profiling agonistic experiments, cells were loaded with $2 \mu \mathrm{M}$ Fluo-4 acetoxymethyl ester (AM) (Invitrogen) for $1 \mathrm{~h}$ in the presence of $0.01 \%$ pluronic acid, washed thrice, and incubated for $30 \mathrm{~min}$ with standard HBSS containing $2.5 \mathrm{mM}$ probenecid and $5 \mathrm{mM}$ HEPES. Compounds to be tested were added at final concentrations ranging from $10^{-11}$ to $10^{-5} \mathrm{M}$ in HBSS, and the fluorescence intensity was measured during $3 \mathrm{~min}$. To evaluate the antagonistic potency of the test compounds, cells were incubated with each compound over 15 min after Fluo-4 AM loading. Then, during fluorescence recording, a pulse of 26RFa was administered at a final concentration of $10^{-7} \mathrm{M}$. After subtraction of the mean fluorescence background, the baseline was normalized to $100 \%$. Fluorescence peak values were determined for each concentration of compound.

\subsection{Statistical Analysis}

Calcium experiments were performed in triplicate, and data, expressed as mean \pm SEM of at least three distinct experiments, were analyzed with the Prism 8.0 software (Graphpad Software, San Diego, CA, USA). $\mathrm{EC}_{50}$ and the $\mathrm{IC}_{50}$ values were determined from concentration-response curves using a sigmoidal dose-response fit with variable slope from at least three independent determinations. Differences between $26 \mathrm{RFa}_{(20-26)}$ and analog activities were analyzed by the Mann-Whitney test. $p$ values $<0.05$ were considered significant.

\subsection{Nomenclature of Targets and Ligands}

All targets and ligands used throughout this manuscript conform with the guidelines outlined by the International Union of Basic and Clinical Pharmacology and British Pharmacological Society (IUPHAR/BPS) Guide to Pharmacology $[5,57]$.

\section{Conclusions}

The C-terminal extremity of 26RFa was previously identified as a pivotal region to modulate its signaling at $h$ QRFPR [36]. In this work, the $\mathrm{Phe}^{22}, \mathrm{Phe}^{24}$ and $\mathrm{Phe}^{26}$ residues of $26 \mathrm{RFa}_{(20-26)}$ were modified using series of point substitutions with natural, side chainconstrained, side chain-modified residues and peptidomimetic building blocks. Subtle chemical modifications in the sequence led to significant improvement in compound potency to activate $h$ QRFPR. As such, a single modification of Phe ${ }^{22}$ with the steric restricted Tic residue decreased the $\mathrm{EC}_{50}$ value from $1640 \pm 259$ to $327 \pm 170 \mathrm{nM}$, providing the most efficient modification at this sequence position. Anchored substituents in the para position of each Phe unit were the most versatile investigated modification. Thereby, the introduction of a para-chloro-phenylalanine in place of the native $\mathrm{Phe}^{24}$ moiety emerged as a favorable replacement. This finding follows the same trend observed in $\left[\mathrm{Pcp}^{26}\right] 26 \mathrm{RFa}_{(20-26)}$, highlighting a limited room to accommodate aromatic residues around the Phe aryl group. Although combination of multiple point-effective modifications does not necessarily translate into an additive or synergic effect, we have explored each position of $26 \mathrm{RFa}_{(20-26)}$ for 
complete optimization of its sequence. Future challenges will be to convert several of these point modifications to low molecular weight 26RFa analogs for metabolic disorder, obesity or diabetes therapies. We are confident that, by utilizing subtle amendments, we can design $26 \mathrm{RFa}_{(20-26)}$-based compounds with nanomolar potency, functional selectivity and in vivo bioavailability.

Author Contributions: B.L. contributed to compound syntheses, acquisition of data, data analysis/interpretation, drafting of the manuscript. K.A., C.N., O.L.M. and C.D. participated in acquisition of data and their analysis/interpretation. J.A.B., J.C., D.V., G.P. and M.P. provided valuable editorial comments and relevant bibliographic references. H.V. and N.C. contributed to critical revision of the manuscript. J.L. conceived and designed the experiments with B.L., K.A. and C.N., contributed to data analysis/interpretation, drafting of the manuscript and approval of the article. All authors have read and agreed to the published version of the manuscript.

Funding: This work was supported by INSERM, the University of Normandy Rouen, the Region Normandy and the European Union. Europe gets involved in Normandy, with European Regional Development Fund (ERDF).

Institutional Review Board Statement: Not applicable.

Informed Consent Statement: Not applicable.

Data Availability Statement: All data are generated during this study.

Conflicts of Interest: The authors declare no competing financial interest.

Sample Availability: Samples of the compounds 1-64 are available from the authors.

\begin{abstract}
Abbreviations
AHPPA: (3S,4S)-4-amino-3-hydroxy-5-phenylpentanoic acid; $\left[\mathrm{Ca}^{2+}\right]_{\mathrm{i}}$, intracellular calcium concentration; Cmpi, 4-carboxymethylpiperazine; Cmpi(2-FPhg), 2-piperazino-2-[(2-fluoro)phenyl]acetic acid; Cmpi(4-FPhg), 2-piperazino-2-[(4-fluoro)phenyl]acetic acid; $h \mathrm{QRFPR}$, human QRFP receptor; 2-Nal, 3-(2-naphtyl)-alanine; NMePhe, $N$-methyl-phenylalanine; NPhe, N-benzyl-glycine; Thi, 3-(2-thienyl)alanine; Oic, octahydroindole-2-carboxylic acid; Phg, phenylglycine; Tic, 1,2,3,4-tetrahydroisoquinoline3-carboxylic acid.
\end{abstract}

\title{
References
}

1. Chartrel, N.; Dujardin, C.; Anouar, Y.; Leprince, J.; Decker, A.; Clerens, S.; Do-Régo, J.-C.; Vandesande, F.; Llorens-Cortes, C.; Costentin, J.; et al. Identification of 26RFa, a hypothalamic neuropeptide of the RFamide peptide family with orexigenic activity. Proc. Natl. Acad. Sci. USA 2003, 100, 15247-15252. [CrossRef]

2. Fukusumi, S.; Yoshida, H.; Fujii, R.; Maruyama, M.; Komatsu, H.; Habata, Y.; Shintani, Y.; Hinuma, S.; Fujino, M. A new peptidic ligand and its receptor regulating adrenal function in rats. J. Biol. Chem. 2003, 278, 46387-46395. [CrossRef]

3. Jiang, Y.; Luo, L.; Gustafson, E.L.; Yadav, D.; Laverty, M.; Murgolo, N.; Vassileva, G.; Zeng, M.; Laz, T.M.; Behan, J.; et al. Identification and characterization of a novel RF-amide peptide ligand for orphan G-protein-coupled receptor SP9155. J. Biol. Chem. 2003, 278, 27652-27657. [CrossRef]

4. Chartrel, N.; Dujardin, C.; Leprince, J.; Desrues, L.; Tonon, M.-C.; Cellier, E.; Cosette, P.; Jouenne, T.; Simonnet, G.; Vaudry, H. Isolation, characterization and distribution of a novel neuropeptide, Rana RFamide (R-RFa), in the brain of the European green frog Rana esculenta. J. Comp. Neurol. 2002, 448, 111-127. [CrossRef]

5. Leprince, J.; Bagnol, D.; Bureau, R.; Fukusumi, S.; Granata, R.; Hinuma, S.; Larhammar, D.; Primeaux, S.; Sopkova-de Oliveira Santos, J.; Tsutsui, K.; et al. The Arg-Phe-amide peptide 26RFa/glutamine RF-amide peptide and its receptor. IUPHAR Review 24. Br. J. Pharmacol. 2017, 174, 3573-3607. [CrossRef] [PubMed]

6. Ukena, K.; Tachibana, T.; Iwakoshi-Ukena, E.; Saito, Y.; Minakata, H.; Kawaguchi, R.; Osugi, T.; Tobari, Y.; Leprince, J.; Vaudry, H.; et al. Identification, localization, and function of a novel avian hypothalamic neuropeptide, 26RFa, and its cognate receptor, $\mathrm{G}$ protein-coupled receptor-103. Endocrinology 2010, 151, 2255-2264. [CrossRef] [PubMed]

7. Tobari, Y.; Iijima, N.; Tsunekawa, K.; Osugi, T.; Haraguchi, S.; Ubuka, T.; Ukena, K.; Okanoya, K.; Tsutsui, K.; Ozawa, H. Identification, localisation and functional implication of 26RFa orthologue peptide in the brain of zebra finch (Taeniopygia guttata). J. Neuroendocrinol. 2011, 23, 791-803. [CrossRef] 
8. Takayasu, S.; Sakurai, T.; Iwasaki, S.; Teranishi, H.; Yamanaka, A.; Williams, S.C.; Iguchi, H.; Kawasawa, Y.I.; Ikeda, Y.; Sakakibara, I.; et al. A neuropeptide ligand of the G protein-coupled receptor GPR103 regulates feeding, behavioral arousal, and blood pressure in mice. Proc. Natl. Acad. Sci. USA 2006, 103, 7438-7443. [CrossRef] [PubMed]

9. Bruzzone, F.; Lectez, B.; Tollemer, H.; Leprince, J.; Dujardin, C.; Rachidi, W.; Chatenet, D.; Baroncini, M.; Beauvillain, J.-C.; Vallarino, M.; et al. Anatomical distribution and biochemical characterization of the novel RFamide peptide 26RFa in the human hypothalamus and spinal cord. J. Neurochem. 2006, 99, 616-627. [CrossRef] [PubMed]

10. Kampe, J.; Wiedmer, P.; Pfluger, P.T.; Castaneda, T.R.; Burget, L.; Mondala, H.; Kerr, J.; Liaw, C.; Oldfield, B.J.; Tschöp, M.H.; et al. Effect of central administration of QRFP(26) peptide on energy balance and characterization of a second QRFP receptor in rat. Brain Res. 2006, 1119, 133-149. [CrossRef]

11. Lectez, B.; Jeandel, L.; El-Yamani, F.-Z.; Arthaud, S.; Alexandre, D.; Mardargent, A.; Jégou, S.; Mounien, L.; Bizet, P.; Magoul, $\mathrm{R}$.; et al. The orexigenic activity of the hypothalamic neuropeptide $26 \mathrm{RFa}$ is mediated by the neuropeptide $\mathrm{Y}$ and proopiomelanocortin neurons of the arcuate nucleus. Endocrinology 2009, 150, 2342-2350. [CrossRef] [PubMed]

12. Do Rego, J.-C.; Leprince, J.; Chartrel, N.; Vaudry, H.; Costentin, J. Behavioral effects of 26RFamide and related peptides. Peptides 2006, 27, 2715-2721. [CrossRef] [PubMed]

13. Moriya, R.; Sano, H.; Umeda, T.; Ito, M.; Takahashi, Y.; Matsuda, M.; Ishihara, A.; Kanatani, A.; Iwaasa, H. RFamide peptide QRFP43 causes obesity with hyperphagia and reduced thermogenesis in mice. Endocrinology 2006, 147, 2916-2922. [CrossRef] [PubMed]

14. Primeaux, S.D.; Blackmon, C.; Barnes, M.J.; Braymer, H.D.; Bray, G.A. Central administration of the RFamide peptides, QRFP-26 and QRFP-43, increases high fat food intake in rats. Peptides 2008, 29, 1994-2000. [CrossRef]

15. Primeaux, S.D.; Barnes, M.J.; Braymer, H.D. Hypothalamic QRFP: Regulation of food intake and fat selection. Horm. Metab. Res. 2013, 45, 967-974. [CrossRef] [PubMed]

16. Zagorácz, O.; Kovács, A.; László, K.; Ollmann, T.; Péczely, L.; Lénárd, L. Effects of direct QRFP-26 administration into the medial hypothalamic area on food intake in rats. Brain Res. Bull. 2015, 118, 58-64. [CrossRef] [PubMed]

17. Navarro, V.M.; Fernández-Fernández, R.; Nogueiras, R.; Vigo, E.; Tovar, S.; Chartrel, N.; Le Marec, O.; Leprince, J.; Aguilar, E.; Pinilla, L.; et al. Novel role of 26RFa, a hypothalamic RFamide orexigenic peptide, as putative regulator of the gonadotropic axis. J. Physiol. 2006, 573, 237-249. [CrossRef]

18. Patel, S.R.; Murphy, K.G.; Thompson, E.L.; Patterson, M.; Curtis, A.E.; Ghatei, M.A.; Bloom, S.R. Pyroglutamylated RFamide peptide 43 stimulates the hypothalamic-pituitary-gonadal axis via gonadotropin-releasing hormone in rats. Endocrinology 2008, 149, 4747-4754. [CrossRef]

19. Palotai, M.; Telegdy, G. Anxiolytic effect of the GPR103 receptor agonist peptide P550 (homolog of neuropeptide 26RFa) in mice. Involvement of neurotransmitters. Peptides 2016, 82, 20-25. [CrossRef] [PubMed]

20. Zagorácz, O.; Ollmann, T.; Péczely, L.; László, K.; Kovács, A.; Berta, B.; Kállai, V.; Kertes, E.; Lénárd, L. QRFP administration into the medial hypothalamic nuclei improves memory in rats. Brain Res. 2020, 1727, 146563. [CrossRef]

21. Yamamoto, T.; Miyazaki, R.; Yamada, T. Intracerebroventricular administration of 26RFa produces an analgesic effect in the rat formalin test. Peptides 2009, 30, 1683-1688. [CrossRef] [PubMed]

22. Yamamoto, T.; Miyazaki, R.; Yamada, T.; Shinozaki, T. Anti-allodynic effects of intrathecally and intracerebroventricularly administered 26RFa, an intrinsic agonist for GRP103, in the rat partial sciatic nerve ligation model. Peptides 2011, 32, 1262-1269. [CrossRef] [PubMed]

23. Yoshida, K.; Nonaka, T.; Nakamura, S.; Araki, M.; Yamamoto, T. Microinjection of 26RFa, an endogenous ligand for the glutamine RF-amide peptide receptor (QRFP receptor), into the rostral ventromedial medulla (RVM), locus coelureus (LC), and periaqueductal grey (PAG) produces an analgesic effect in rats. Peptides 2019, 115, 1-7. [CrossRef]

24. Gouardères, C.; Mazarguil, H.; Mollereau, C.; Chartrel, N.; Leprince, J.; Vaudry, H.; Zajac, J.-M. Functional differences between NPFF1 and NPFF2 receptor coupling: High intrinsic activities of RFamide-related peptides on stimulation of [ $\left.{ }^{35} \mathrm{~S}\right] \mathrm{GTP} \gamma \mathrm{S}$ binding. Neuropharmacology 2007, 52, 376-386. [CrossRef]

25. Chartrel, N.; Alonzeau, J.; Alexandre, D.; Jeandel, L.; Alvear-Perez, R.; Leprince, J.; Boutin, J.; Vaudry, H.; Anouar, Y.; LlorensCortes, C. The RFamide neuropeptide 26RFa and its role in the control of neuroendocrine functions. Front. Neuroendocrinol. 2011, 32, 387-397. [CrossRef]

26. Prévost, G.; Jeandel, L.; Arabo, A.; Coëffier, M.; El Ouahli, M.; Picot, M.; Alexandre, D.; Gobet, F.; Leprince, J.; Berrahmoune, H.; et al. Hypothalamic neuropeptide 26RFa acts as an incretin to regulate glucose homeostasis. Diabetes 2015, 64, $2805-2816$. [CrossRef]

27. Prévost, G.; Arabo, A.; Le Solliec, M.-A.; Bons, J.; Picot, M.; Maucotel, J.; Berrahmoune, H.; El Mehdi, M.; Cherifi, S.; Benani, A.; et al. Neuropeptide 26RFa (QRFP) is a key regulator of glucose homeostasis and its activity is markedly altered in obese/hyperglycemic mice. Am. J. Physiol. Endocrinol. Metab. 2019, 317, E147-E157. [CrossRef]

28. Prévost, G.; Picot, M.; Le Solliec, M.-A.; Arabo, A.; Berrahmoune, H.; El Mehdi, M.; Cherifi, S.; Benani, A.; Nédélec, E.; Gobet, F.; et al. The neuropeptide 26RFa in the human gut and pancreas: Potential involvement in glucose homeostasis. Endocr. Connect. 2019, 8, 941-951. [CrossRef] [PubMed]

29. El-Mehdi, M.; Takhlidjt, S.; Khiar, F.; Prévost, G.; Do Rego, J.-L.; Do Rego, J.-C.; Benani, A.; Nedelec, E.; Godefroy, D.; Arabo, A.; et al. Glucose homeostasis is impaired in mice deficient in the neuropeptide 26RFa (QRFP). BMJ Open Diabetes Res. Care 2020, 8, e000942. [CrossRef] [PubMed] 
30. Quillet, R.; Ayachi, S.; Bihel, F.; Elhabazi, K.; Ilien, B.; Simonin, F. RF-amide neuropeptides and their receptors in Mammals: Pharmacological properties, drug development and main physiological functions. Pharmacol. Ther. 2016, 160, 84-132. [CrossRef]

31. Mazarguil, H.; Gouardères, C.; Tafani, J.A.; Marcus, D.; Kotani, M.; Mollereau, C.; Roumy, M.; Zajac, J.M. Structure-activity relationships of neuropeptide FF: Role of C-terminal regions. Peptides 2001, 22, 1471-1478. [CrossRef]

32. Boyle, R.G.; Downham, R.; Ganguly, T.; Humphries, J.; Smith, J.; Travers, S. Structure-activity studies on prolactin-releasing peptide (PrRP). Analogues of PrRP-(19-31)-peptide. J. Pept. Sci. 2005, 11, 161-165. [CrossRef]

33. Orsini, M.J.; Klein, M.A.; Beavers, M.P.; Connolly, P.J.; Middleton, S.A.; Mayo, K.H. Metastin (KiSS-1) mimetics identified from peptide structure-activity relationship-derived pharmacophores and directed small molecule database screening. J. Med. Chem. 2007, 50, 462-471. [CrossRef] [PubMed]

34. Gicquel, S.; Mazarguil, H.; Desprat, C.; Allard, M.; Devillers, J.P.; Simonnet, G.; Zajac, J.M. Structure-activity study of neuropeptide FF: Contribution of N-terminal regions to affinity and activity. J. Med. Chem. 1994, 37, 3477-3481. [CrossRef]

35. Mazarguil, H.; Mollereau, C.; Czaplicki, G.; Zajac, J.M. Study of the N-terminal part of peptidic selective NPFF 2 agonists. Peptides 2012, 37, 157-160. [CrossRef] [PubMed]

36. Le Marec, O.; Neveu, C.; Lefranc, B.; Dubessy, C.; Boutin, J.A.; Do-Régo, J.-C.; Costentin, J.; Tonon, M.-C.; Tena-Sempere, M.; Vaudry, H.; et al. Structure-activity relationships of a series of analogues of the RFamide-related peptide 26RFa. J. Med. Chem. 2011, 54, 4806-4814. [CrossRef]

37. Neveu, C.; Lefranc, B.; Tasseau, O.; Do Rego, J.-C.; Bourmaud, A.; Chan, P.; Bauchat, P.; Le Marec, O.; Chuquet, J.; Guilhaudis, L.; et al. Rational design of a low molecular weight, stable, potent, and long-lasting GPR103 aza- $\beta^{3}$-pseudopeptide agonist. J. Med. Chem. 2012, 55, 7516-7524. [CrossRef]

38. Alim, K.; Lefranc, B.; Sopkova-de Oliveira Santos, J.; Dubessy, C.; Picot, M.; Boutin, J.A.; Vaudry, H.; Chartrel, N.; Vaudry, D.; Chuquet, J.; et al. Design, synthesis, molecular dynamics simulation and functional evaluation of a novel series of 26RFa peptide analogues containing a mono- or polyalkyl guanidino arginine derivative. J. Med. Chem. 2018, 61, 10185-10197. [CrossRef]

39. Pierry, C.; Couve-Bonnaire, S.; Guilhaudis, L.; Neveu, C.; Marotte, A.; Lefranc, B.; Cahard, D.; Segalas-Milazzo, I.; Leprince, J.; Pannecoucke, X. Fluorinated pseudopeptide analogues of neuropeptide 26RFa: Synthesis, biological and structural studies. ChemBioChem. 2013, 14, 1620-1633. [CrossRef]

40. Rouméas, L.; Humbert, J.P.; Schneider, S.; Doebelin, C.; Bertin, I.; Schmitt, M.; Bourguignon, J.J.; Simonin, F.; Bihel, F. Effects of systematic N-terminus deletions and benzoylations of endogenous RF-amide peptides on NPFF1R, NPFF2R, GPR10, GPR54 and GPR103. Peptides 2015, 71, 156-161. [CrossRef] [PubMed]

41. Anjana, R.; Vaishnavi, M.K.; Sherlin, D.; Kumar, S.P.; Naveen, K.; Kanth, P.S.; Sekar, K. Aromatic-aromatic interactions in structures of proteins and protein-DNA complexes: A study based on orientation and distance. Bioinformation 2012, 8, 1220-1224. [CrossRef]

42. Makwana, K.M.; Mahalakshmi, R. Implications of aromatic-aromatic interactions: From protein structures to peptide models. Protein Sci. 2015, 24, 1920-1933. [CrossRef] [PubMed]

43. de Freitas, R.F.; Schapira, M. A systematic analysis of atomic protein-ligand interactions in the PDB. MedChemComm 2017, 8 , 1970-1981. [CrossRef]

44. Meyer, E.A.; Castellano, R.K.; Diederich, F. Interactions with aromatic rings in chemical and biological recognition. Angew. Chem. Int. Ed. Engl. 2003, 42, 1210-1250. [CrossRef] [PubMed]

45. Neveu, C.; Dulin, F.; Lefranc, B.; Galas, L.; Calbrix, C.; Bureau, R.; Rault, S.; Chuquet, J.; Boutin, J.A.; Guilhaudis, L.; et al. Molecular basis of agonist docking in a human GPR103 homology model by site-directed mutagenesis and structure-activity relationship studies. Br. J. Pharmacol. 2014, 171, 4425-4439. [CrossRef]

46. Kotha, S.; Deodhar, D.; Khedkar, P. Diversity-oriented synthesis of medicinally important 1,2,3,4-tetrahydroisoquinoline-3carboxylic acid (Tic) derivatives and higher analogs. Org. Biomol. Chem. 2014, 12, 9054-9091. [CrossRef]

47. Williams, P.G.; Yoshida, W.Y.; Moore, R.E.; Paul, V.J. The isolation and structure elucidation of Tasiamide B, a 4-amino-3-hydroxy5-phenylpentanoic acid containing peptide from the marine Cyanobacterium symploca sp. J. Nat. Prod. 2003, 66, $1006-1009$. [CrossRef] [PubMed]

48. Fauchère, J.L.; Thurieau, C. Evaluation of the stability of peptides and pseudopeptides as a tool in peptide drug design. Adv. Drug Res. 1992, 23, 127-158.

49. Thuau, R.; Guilhaudis, L.; Ségalas-Milazzo, I.; Chartrel, N.; Oulyadi, H.; Boivin, S.; Fournier, A.; Leprince, J.; Davoust, D.; Vaudry, H. Structural studies on 26RFa, a novel human RFamide-related peptide with orexigenic activity. Peptides 2005, 26, 779-789. [CrossRef]

50. Butterfield, S.M.; Patel, P.R.; Waters, M.L. Contribution of aromatic interactions to $\alpha$-helix stability. J. Am. Chem. Soc. 2002, 124, 9751-9755. [CrossRef]

51. Mosberg, H.I.; Hurst, R.; Hruby, V.J.; Gee, K.; Yamamura, H.I.; Galligan, J.J.; Burks, T.F. Bis-penicillamine enkephalins possess highly improved specificity toward delta opioid receptors. Proc. Natl. Acad. Sci. USA 1983, 80, 5871-5874. [CrossRef]

52. Marcotte, I.; Separovic, F.; Auger, M.; Gagné, S.M. A multidimensional 1H NMR investigation of the conformation of methionineenkephalin in fast-tumbling bicelles. Biophys. J. 2004, 86, 1587-1600. [CrossRef]

53. Datta, S.; Shamala, N.; Banerjee, A.; Balaram, P. Conformational variability of Gly-Gly segments in peptides: A comparison of the crystal structures of an acyclic pentapeptide and an octapeptide. Biopolymers 1997, 41, 331-336. [CrossRef] 
54. Touchard, A.; Aili, S.R.; Téné, N.; Barassé, V.; Klopp, C.; Dejean, A.; Kini, R.M.; Mrinalini; Coquet, L.; Jouenne, T.; et al. Venom peptide repertoire of the European myrmicine ant Manica rubida: Identification of insecticidal toxins. J. Proteome Res. 2020, 19, 1800-1811. [CrossRef] [PubMed]

55. Dubessy, C.; Cartier, D.; Lectez, B.; Bucharles, C.; Chartrel, N.; Montero-Hadjadje, M.; Bizet, P.; Chatenet, D.; Tostivint, H.; Scalbert, E.; et al. Characterization of urotensin II, distribution of urotensin II, urotensin II-related peptide and UT receptor mRNAs in mouse: Evidence of urotensin II at the neuromuscular junction. J. Neurochem. 2008, 17, 361-374. [CrossRef] [PubMed]

56. Gutiérrez-Pascual, E.; Leprince, J.; Martínez-Fuentes, A.J.; Ségalas-Milazzo, I.; Pineda, R.; Roa, J.; Duran-Prado, M.; Guilhaudis, L.; Desperrois, E.; Lebreton, A.; et al. In vivo and in vitro structure-activity relationships and structural conformation of kisspeptin-10-related peptides. Mol. Pharmacol. 2009, 76, 58-67. [CrossRef]

57. Alexander, S.P.H.; Christopoulos, A.; Davenport, A.P.; Kelly, E.; Mathie, A.; Peters, J.A.; Veale, E.L.; Armstrong, J.F.; Faccenda, E.; Harding, S.D.; et al. The concise guide to pharmacology 2019/20: G protein-coupled receptors. Br. J. Pharmacol. 2019, 176, S21-S141. [CrossRef] [PubMed] 